\title{
Thermal electron anisotropy driven by kinetic Alfúen waves in the Earth's magnetotail
}

\author{
Alexander Lukin ${ }^{1,2}$, Anton Artemyev ${ }^{3,1}$, Evgeny Panov ${ }^{4}$, Rumi Nakamura ${ }^{4}$, Anatoly Petrukovich ${ }^{1}$, \\ Robert Ergun ${ }^{5}$, Barbara Giles ${ }^{6}$, Yuri Khotyaintsev ${ }^{7}$, Per Arne Lindqvist ${ }^{8}$, Christopher Russell $^{3}$, and \\ Robert Strangeway ${ }^{3}$ \\ ${ }^{1}$ Space Research Institute, RAS, Moscow, Russia. \\ ${ }^{2}$ Faculty of Physics, National Research University Higher School of Economics, Moscow, Russia. \\ ${ }^{3}$ Institute of Geophysics and Planetary Physics, University of California, Los Angeles, USA. \\ ${ }^{4}$ Space Research Institute, Austrian Academy of Sciences, Graz, Austria. \\ ${ }^{5}$ Laboratory for Atmospheric and Space Physics, University of Colorado Boulder, Boulder, CO, USA. \\ ${ }^{6}$ NASA Goddard Space Flight Center, Greenbelt, MD, USA. \\ ${ }^{7}$ Swedish Institute of Space Physics, Uppsala, Sweden. \\ ${ }^{8}$ Division of space and plasma physics, KTH Royal Institute of Technology, Stockholm, Sweden.
}

Correspondence: A. Lukin (as.lukin.phys@gmail.com)

\begin{abstract}
Thermal and subthermal electron populations in the Earth's magnetotail are usually characterized by pronounced field-aligned anisotropy that contributes to generation of strong electric currents within the magnetotail current sheet. Formation of this anisotropy requires electron field-aligned acceleration, and thus likely involves field-aligned electric fields. Such fields can be carried by various electromagnetic waves generated by fast plasma flows interacting with ambient magnetotail plasma.

5 In this paper we consider one of the most intense observed wave emissions, kinetic Alfv́en waves, that often accompany fast plasma flows in the magnetotail. Using two tail seasons $(2017,2018)$ of MMS observations we have collected statistics of 80 fast plasma flows (or bursty bulk flows) events with distinctive enhancement of intensity of broadband electromagnetic waves (kinetic Alfv́en waves). We show correlation the intensity of electric fields of kinetic Alfv́en waves and characteristics of electron anisotropy distributions: the parallel electron anisotropy increases with magnitude of the wave parallel electric field. Also the energy range of this electron anisotropic population is well within the expected acceleration range for assumed kinetic Alfv́en waves. Our results indicate an important role of KAWs in production of thermal field-aligned electron population typically observed in the Earth's magnetotail.
\end{abstract}

\section{Introduction}

Electron population of the Earth's magnetotail plays an important role in magnetotail current sheet structure and dynamics. Being mostly magnetized (in contrast to well scattered and isotropic ions, e.g. Walsh et al. (2011); Wang et al. (2013)) electron 
population can be quite anisotropic (Walsh et al., 2011; Artemyev et al., 2014), and such anisotropy in the high- $\beta$ plasma sheet may be significant source of plasma currents (Hau, 1993; Artemyev et al., 2020; Kamaletdinov et al., 2020). These currents contribute to both the cross-field plasma sheet current flowing in the dawn-dusk direction (e.g., Zelenyi et al., 2011; Artemyev et al., 2016; Mingalev et al., 2018) and to field-aligned currents (Walsh et al., 2013; Artemyev et al., 2017) contributing to magnetosphere-ionosphere coupling.

There are two distinct types of electron anisotropic populations in the Earth's magnetotail. In the dipolarized magnetotail (e.g., at dipolarization fronts and behind them) the thermal $(<10 \mathrm{keV})$ electron population is predominantly transversely anisotropic (with $T_{e \perp}>T_{e \|}$; see Khotyaintsev et al. (2011); Fu et al. (2012); Runov et al. (2013); Zhang et al. (2018)), what results in whistler-mode wave generation (Khotyaintsev et al., 2011; Panov et al., 2013; Le Contel et al., 2009; Breuillard et al., 2016; Grigorenko et al., 2020). This anisotropy is due to electron betatron heating at the dipolarization front (Fu et al., 2013; Birn et al., 2012, 2013), and thus observations of such transversely anisotropic electrons are quite transient. In the quiet plasma sheet with stretched magnetic field lines the thermal electron population is predominantly field-aligned anisotropic (with $T_{e \|}>T_{e \perp}$; see Hada et al. (1981); Stiles et al. (1978); Artemyev et al. (2014)). The strongest patterns of such fieldaligned anisotropic electrons, electron beams, contribute to electrostatic waves and nonlinear electron holes widely observed at plasma sheet boundary layers (Matsumoto et al., 1994; Tong et al., 2018). Origins of such anisotropy are not yet revealed. Adiabatic acceleration during magnetotail dipolarization may, in principle, contribute to field-aligned anisotropy (Sergeev et al., 2001; Birn et al., 2014), but adiabatic heating during magnetotail convection should mostly contribute to transverse anisotropy (Lyons, 1984; Zelenyi et al., 2013). The perspective mechanisms for field-aligned electron anisotropy generation are ionospheric outflow (Walsh et al., 2013; Khazanov et al., 2014) and electron acceleration by field-aligned electric fields. Such fields are carried by broadband electrostatic noise dominated by electron holes and solitary waves (Mozer et al., 2015; Vasko et al., 2017b; Malaspina et al., 2018) and by kinetic Alfv́en waves (Chaston et al., 2012, 2014; Ergun et al., 2015; Stawarz et al., 2017). Although, electron holes and solitary waves can shape field-aligned electron populations (Vasko et al., 2017a), their generation results from the relaxation of electron beams (Omura et al., 1996; Goldman et al., 1999; Lotekar et al., 2020), i.e. electron field-aligned anisotropy should be origin of such nonlinear structures. Kinetic Alfv́en waves are predominantly generated around energy release regions (e.g., magnetic reconnection, see Liang et al., 2016) and effectively accelerate electrons along magnetic field lines (Damiano et al., 2015; Damiano et al., 2016; Artemyev et al., 2015). Thus, these waves transfer ion kinetic energy to electron heating and can be responsible for generation of field-aligned electron anisotropy.

Kinetic Alfv́en waves (KAWs) are widely observed (Chaston et al., 2014; Ergun et al., 2015) and modeled (Lin et al., 2017; Sharma Pyakurel et al., 2018; Cheng et al., 2020) in the plasma sheet, and their dispersion guarantees a finite fieldaligned electric field (Stasiewicz et al., 2000), that potentially can accelerate plasma sheet electrons along magnetic field lines. Although the typical KAW phase speed (about Alfv́en speed, that is $v_{A} \sim 100-500 \mathrm{~km} / \mathrm{s}$ in the plasma sheet) is insufficiently high for Landau resonance with plasma sheet electrons $\left(m_{e} v_{A}^{2} / 2<1 \mathrm{eV}\right)$, the large effective wave potential $\sim E_{\|} / k_{\|} \sim 1 \mathrm{keV}$ (Damiano et al., 2015; Artemyev et al., 2015; Wang et al., 2019a) results in resonance widening (Palmadesso, 1972; Karimabadi et al., 1990). Electrons fast bouncing along magnetic field line can be trapped into such potential (Watt and Rankin, 2009) or reflect from it (Kletzing, 1994), and both effects would result in electron heating along magnetic field lines. However, so far 
there were no statistical evidence of KAWs contribution to field-aligned electron anisotropy in the plasma sheet. In this study we use observations of Magnetospheric Multiscale (MMS) Mission (Burch et al., 2016) in the Earth's magnetotail to confirm the key KAWs' role in shaping plasma sheet electron anisotropy.

Following Chaston et al. (2012), we consider bursty bulk flows (Baumjohann et al., 1990; Angelopoulos et al., 1992) as a main source of KAWs. For statistics of such flows collected in 2017-2018 MMS tail seasons, we identify KAWs from their dispersion properties (e.g. Chaston et al., 2014) and investigate correlations of KAWs characteristics and thermal electron anisotropy.

\section{Spacecraft instruments and dataset}

60 We focus on the KAW signatures associated with the fast plasma flows in the Earth's magnetotail at radial distances of $\sim$ $15-30 R_{E}$. We pick up all intervals lasting more than 10 minutes and associated with increased plasma bulk velocities. Then we compare observed magnetic and electric field spectra with the theoretical predictions for KAW (see details below) and keep in dataset only intervals that are characterized by:

- plasma flow component along Earth-Sun line GSE $V_{x}>100 \mathrm{~km} / \mathrm{s}$ during interval

- spacecraft are near the neutral plane (mean value of the GSE $\left|B_{x}\right| \leq 10 n T$ )

- Observed wave field spectra are close to theoretical predictions

For two tail seasons $(2017,2018)$ we have selected 81 events (see spatial distribution on Fig. 1 and two typical examples on Figs. 3 and 4).

We use burst-mode fields and plasma data measured on board MMS1 spacecraft (Burch et al., 2016): magnetic field data with $8 \mathrm{~ms}$ time resolution from the fluxgate magnetometer (Russell et al., 2016); electric field data with 1ms time resolution from Spin-plane Double Probe instrument/Axial Double Probe instrument(SDP/ADP) (Ergun et al., 2016; Lindqvist et al., 2016; Torbert et al., 2016); plasma moments, energy and pitch-angle distributions from the Fast Plasma Investigation (FPI) (Pollock et al., 2016) with 150ms and 30ms for ions and electrons, respectively. Hereinafter in our calculations we use the field-aligned coordinate system: the parallel direction $(\|)$ is along background magnetic field vector (we use low-pass filtering with the cutoff frequency of $0.01 \mathrm{~Hz}$ ), the first perpendicular component $\left(\perp_{1}\right)$ is along ion bulk velocity component perpendicular to the background magnetic field, and the second perpendicular component $\left(\perp_{2}\right)$ completes the right-hand system. We subtract background magnetic field from the measured magnetic field vector to estimate $>0.01 \mathrm{~Hz}$ magnetic field perturbations (see example on Fig. 2). The same procedure is applied to the electric field vector. We also construct continuous wavelet spectra (using Morlet wavelets) of the parallel and perpendicular components of magnetic and electric field perturbations.

In the rest reference frame KAWs have frequencies less than the ion cyclotron frequency $\omega_{i}$, whereas plasma flow speed in our study usually exceeds $100 \mathrm{~km} / \mathrm{s}$ (with mean value of $\sim 400 \mathrm{~km} / \mathrm{s}$ ). Thus, we expect that in the spacecraft frame all the spectrum properties will be dominated by the Doppler shift, i.e. $\omega_{s c}=\omega+\boldsymbol{k} \cdot \boldsymbol{v} \gg \omega$ and the upper frequency limit of the interest will be well higher than $\omega_{i}$. So in our analysis we consider wave perturbations with frequencies up to the lower hybrid 


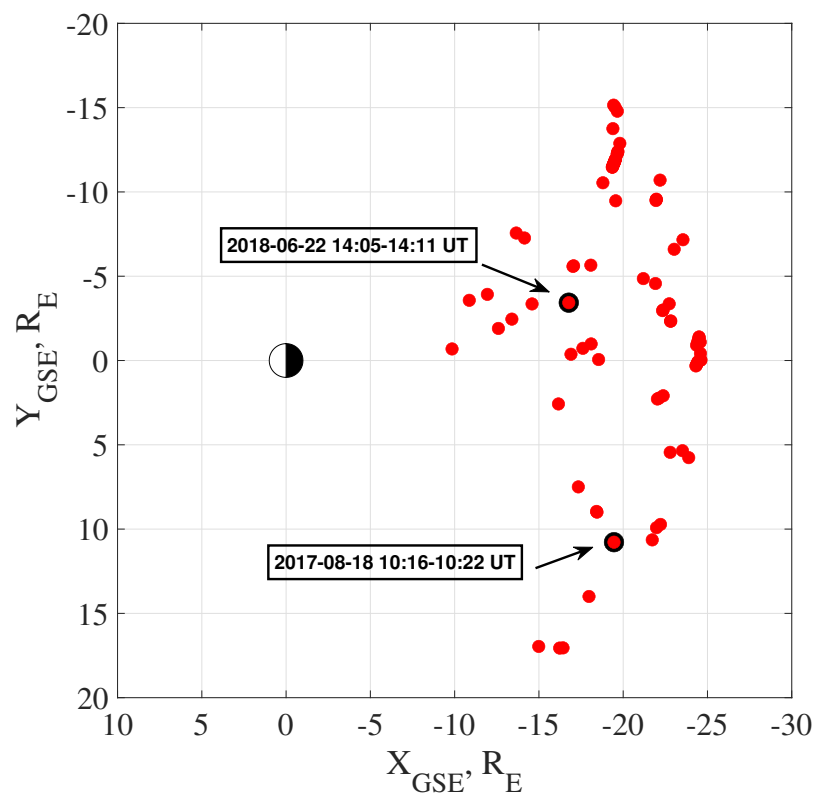

Figure 1. Coordinates of all events.

frequency (see details below). It has been shown (see Stasiewicz et al., 2000; Chaston et al., 2012) that for warm plasma in the low frequency limit $\left(\omega \ll \omega_{i}\right)$ KAWs show following polarization properties:

$\frac{E_{\perp}}{B_{\perp}}=\frac{v_{A}\left(1+k_{\perp}^{2} \rho_{i}^{2}\right)}{\sqrt{1+k_{\perp}^{2}\left(\rho_{i}^{2}+\rho_{s}^{2}\right)}}$

and

$\frac{B_{\|}}{\sqrt{B_{\perp 1}^{2}+B_{\perp 2}^{2}}}=i \beta \frac{k_{\perp} v_{A}}{\omega_{i}} \frac{1}{\sqrt{1+k_{\perp}^{2}\left(\rho_{i}^{2}+\rho_{s}^{2}\right)}}$

where $v_{A}$ is local Alfv́en speed, $\rho_{i}$ is ion gyroradius, $\rho_{s}$ is ion acoustic gyroradius, $k_{\perp}$ is the transverse component of the wave vector $\boldsymbol{k}, \beta=4 \pi n\left(T_{e}+T_{i}\right) / B_{0}^{2}, B_{0}$ is the magnitude of background magnetic field, $B_{\perp 1}$ and $B_{\perp 2}$ are two transverse wave magnetic field components.

Note that we deal with quite inhomogeneous plasma and magnetic fields of fast plasma flows, but we use a local dispersion relation of KAWs (Chaston et al., 2012) and omit the effects of plasma gradients (Bashir et al., 2019) and nonlocal wave properties (Huang et al., 2018; Wang et al., 2019b). These effects can result in deviation of theoretical dispersion predictions from the observed wave characteristics. To estimate a possible role of plasma inhomogeneity we evaluate theoretical equations both for local plasma parameters and for parameters at some distance from the equatorial plane. We use the vertical stress balance condition $p=B^{2} / 8 \pi+n\left(T_{i}+T_{e}\right)=$ const and definition of the magnetotail lobe pressure $p=B_{\text {lobe }}^{2} / 8 \pi$ to determine magnetic fields at the distance where plasma pressure is $30 \%$ of the equatorial value: $B_{\text {middle }}^{2} / 8 \pi=0.7 p, n\left(T_{i}+T_{e}\right)_{\text {middle }}=$ 

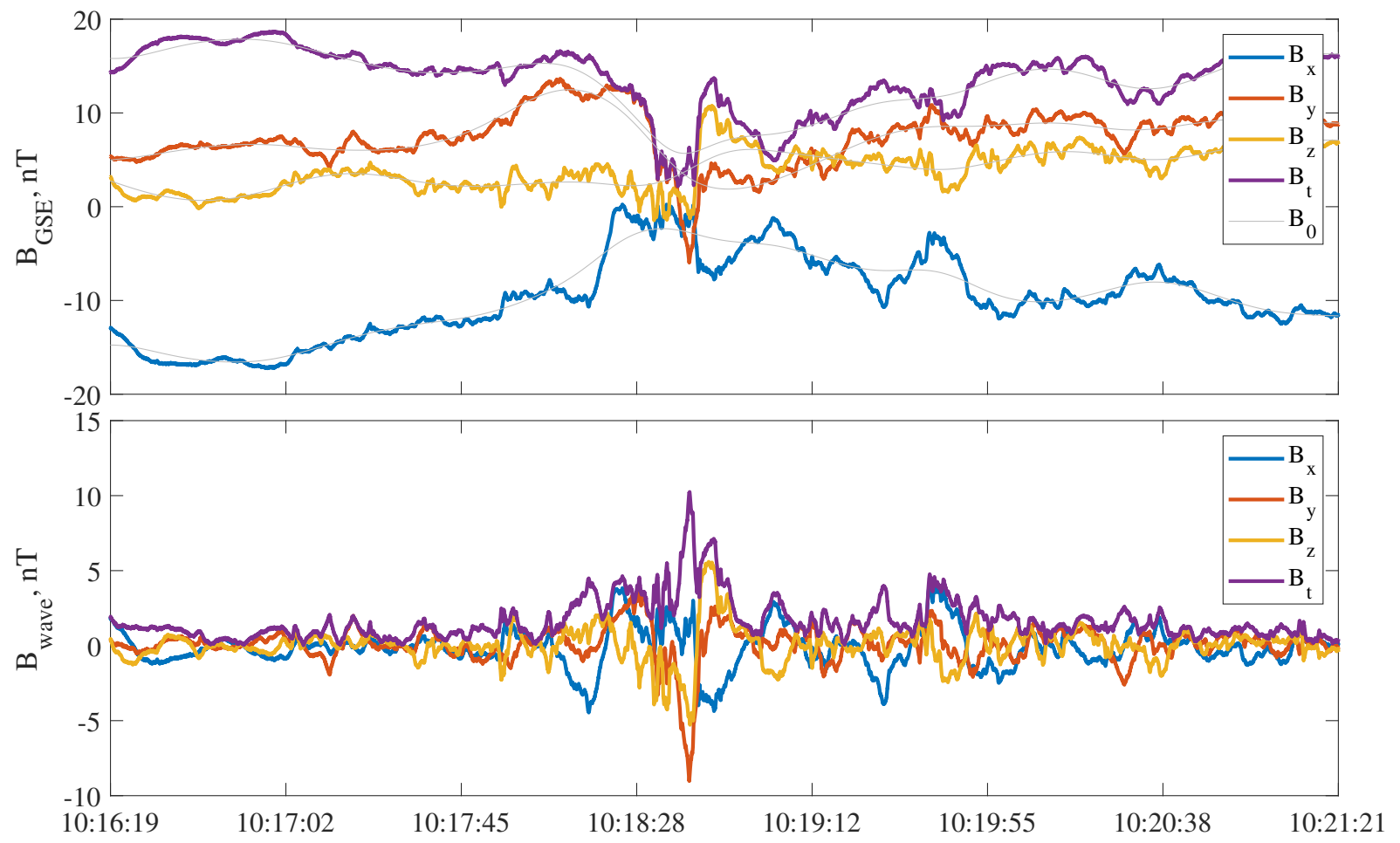

Figure 2. Wave (perturbation) magnetic field determination: gray lines show low-pass filtered background field.

$0.3 p$. We additionally assume $n=$ const (based on statistics of current sheets in the middle tail, see Runov et al. (2006)) and

$T_{e} / T_{i}=$ const (based on comparison of $T_{e}\left(B_{x}\right)$ and $T_{i}\left(B_{x}\right)$ profiles in the middle tail, see Artemyev et al. (2011b, a)). Thus, we provide theoretical estimates for $B_{\|} / \sqrt{B_{\perp 1}^{2}+B_{\perp 2}^{2}}$ at some distance from the equatorial plane together with estimates for local plasma parameters (see yellow curve in the Fig. 5, 6). Estimates at some distance from equatorial plane are used only to show the range of theoretical expectations for wave dispersion across the plasma sheet.

We aim at statistically analyzing the relation of electron flux (thermal) anisotropy and KAW electric field intensity. Such analysis should reveal a possible KAW role in shaping of the anisotropic electron populations. For such statistical comparison, we consider normalized electron energy spectra and electric field spectra. We use mean absolute value of convectional electric field $\left|\boldsymbol{V}_{\boldsymbol{i}} \times \boldsymbol{B}_{\mathbf{0}}\right|$ for electric field magnitude normalization, mean value of electron temperature $T_{e}$ and mean absolute value of KAW electrostatic potential $|\phi|$ for electron energy normalization (averaging is performed for the MMS1 probe within the time interval of enhanced ion bulk velocity; $\left|V_{x}\right|>200 \mathrm{~km} / \mathrm{s}$, typically lasting for $\sim 5$ minutes). To estimate $\phi$ we consider the 
$\phi(t)=-\operatorname{Im}\left(\int_{-\infty}^{\infty} \frac{E_{\|}(\omega)}{k_{\|}(\omega)} e^{i \omega t} \frac{d \omega}{2 \pi}\right)$

Thus, knowing the transverse component $k_{\perp}$ and wave frequency in the spacecraft frame $\omega$, we estimate the parallel $k_{\|}$from equation (Chaston et al., 2008):

$k_{\|}^{2} v_{A}^{2} k_{\perp}^{2} \rho_{s}^{2}=\left(\omega^{2}-\frac{k_{\|}^{2} v_{A}^{2}}{\xi_{i}^{2}}\right)\left(1-\frac{k_{\|}^{2} v_{s}^{2}}{\omega^{2}} I_{0}\left(\alpha_{i}\right) e^{-\alpha_{i}}\right)$

where $\alpha_{i}=k_{\perp}^{2} \rho_{i}^{2}, \xi_{i}=\left(1-I_{0}\left(\alpha_{i}\right) e^{-\alpha_{i}}\right) / \alpha_{i}, v_{s}$ is the ion acoustic speed and $I_{0}$ is the zeros order modified Bessel function.

\section{Case-study}

We start with two example events from our dataset shown in Figs. 3, 4. Both events are characterized by enhanced electric and magnetic field fluctuations and strong plasma flow with $V_{i} \sim 500 \mathrm{~km} / \mathrm{s}$. There is magnetic field dipolization front with $B_{z}$ increase (Runov et al., 2009; Nakamura et al., 2001) at $\sim 18: 18: 20$ for the first event and at $\sim 14: 07: 00$ for the second one. The dipolization front separates two electron populations with quite different pitch-angle distributions and energy spectra (the sharper difference is seen for the second event); e.g. for the $[0.2,2] \mathrm{keV}$ energy range (panel (f) of Fig. 4) the pitch-angle distribution becomes dominated by field-aligned flows behind the front. Panel (h) in Fig. 4 shows energy distribution of flux anisotropy calculated as

$A_{\perp}=\frac{F_{\perp}-F_{\|}}{F_{\perp}+F_{\|}}$

where $F_{\|}=\max \left(F_{\text {parallel }}, F_{\text {anti }}\right)$,

$\left\{\begin{array}{l}F_{\text {parallel }}: \alpha \in\left[0^{\circ}, 18^{\circ}\right] ; \\ F_{\text {anti }}: \alpha \in\left[162^{\circ}, 180^{\circ}\right] ; \\ F_{\perp}: \alpha \in\left[72^{\circ}, 108^{\circ}\right] ;\end{array}\right.$

and $\alpha$ is pitch-angle. We use SPEDAS software (Angelopoulos et al., 2019) to calculate $F_{\perp}, F_{\|}$fluxes (note FPI pitch-angle resolution is $6^{\circ}$ ). The energy range of $[0, \sim 200] \mathrm{eV}$ is contaminated by photoelectrons. To exclude this population from the consideration, we use electron data only for $>100 \mathrm{eV}$ energy range.

Figure 4 shows parameters of magnetic and electric field perturbations in the field-aligned coordinate system after subtraction of the low-pass filtered background field. Black vertical lines here bound time intervals which we use for spectral analysis. Previous investigations of KAWs with Cluster (Escoubet et al., 2001) and THEMIS (Angelopoulos, 2008) measurements (Chaston et al., 2005, 2008, 2012, 2013) rely on theoretical relations that provide an estimate of the parallel electric field component from the measured perpendicular one. In our work we use direct measurements of the parallel electric component available on MMS (Torbert et al., 2016; Ergun et al., 2016). 
There is a clear enhancement of the wave activity in the wide frequency range for the electric field components (see panels $(\mathrm{d}, \mathrm{e})$ ) and mainly up to ion cyclotron frequency for the magnetic field components (see panels (f,g)). Time-averaged wave spectra are shown in Figs. 5, 6: up to the $\sim 0.1 \mathrm{~Hz}$ magnetic and electric spectral densities (transverse components) have almost the same profiles (left panels of Figs. 5, 6), whereas $E_{\perp} / B_{\perp} \sim$ const $\sim v_{A}$, i.e. we deal with Alfv́enic perturbations. The ratio $E_{\perp} / B_{\perp}$ grows with frequency, as it is expected for KAWs (Chaston et al., 2012). Panel (b) compares observed ratio $E_{\perp} / B_{\perp}$ and predicted the from the KAW's dispersion relation: up to the lower-hybrid frequency observations follow the theoretical model quite well, and for frequencies larger than the lower-hybrid frequency we likely deal with another wave mode (e.g., lower-hybrid drift waves or whistler-mode waves often observed around the dipolarization fronts, see Le Contel et al. (2009); Deng et al. (2010); Khotyaintsev et al. (2011); Panov et al. (2013); Grigorenko et al. (2020); Wendel et al. (2019); Huang et al. (2016)). Thus, we consider electric and magnetic wave characteristics only below the lower-hybrid frequency.

At the same time, some compressional modes are presented in the ultra-low frequency range $\left(B_{\|} / B_{\perp}>1\right)$. Most likely KAWs are mixed with some ultra-low frequency compressional (diamagnetic perturbations) that are typical for high plasma pressure magnetotail (Volwerk et al., 2003; Runov et al., 2014). Observed frequencies of such perturbations are dominated by the Doppler shift and we cannot distinguish them from KAWs. However, contrast to KAWs, ultra-low frequency diamagnetic perturbations are MHD modes that are not expected to carry any significant field-aligned fields. Thus, we can assume that electron field-aligned acceleration (if any) is associated with observed KAWs, that are well seen in $E_{\perp} / B_{\perp}$ dispersion for the intermediate frequency range.

Figures 5, 6 demonstrate that observed wave activity can be associated with KAWs in the range of $\left.\sim 10^{-2}, 10^{1}\right] \mathrm{Hz}$, up to the lower-hybrid frequency. However, there are likely additional compressional (with larger $B_{\|}$) low frequency modes that contribute to the observed spectra around $\sim 10^{-2} \mathrm{~Hz}$. Panels (c) show that KAWs dispersion underestimate $B_{\|} / B_{\perp}$ for low frequencies and slightly overestimate it for $\sim\left[10^{-1}, 10^{1}\right] \mathrm{Hz}$ frequency range. We also plot theoretical curve for plasma and magnetic field parameters corresponding to some distance from the equatorial plane (see details above), and the observed $B_{\|} / B_{\perp}$ values are between these two theoretical curves. Thus, although difference between observed $B_{\|} / B_{\perp}$ and theoretical predictions for KAWs in $\sim\left[10^{-1}, 10^{1}\right] \mathrm{Hz}$ frequency range can be explained by strong variation of plasma characteristics across the plasma sheet, KAWs theory underestimates compressional magnetic fields at lower frequencies, where observations are likely dominated by diamagnetic fluctuations typical for diplarization fronts (Runov et al., 2014) and fast plasma flows (Bauer et al., 1995; Panov et al., 2012; Volwerk et al., 2004a).

To reveal the relation between KAWs' electric fields and electron anisotropy, we consider 2D distributions of flux anisotropy in energy and electric field space. For this we bin a field amplitude range and average all energy distributions over the time interval bounded by black vertical lines on Figs. 3, 4 within the same bin of field amplitude shown in Figs. 7, 8. There are three distinguishing energy ranges with different anisotropy (note we normalize energy to instantaneous electron temperature measurements):

1. $E / T_{e} \lesssim 10^{-1}$

2. $10^{-1} \lesssim E / T_{e} \lesssim 2-4$ 
The first (lowest) range can be filled by transversely anisotropic electrons of unknown origin (this could be an effect of real transverse acceleration of cold electrons at dipolarization fronts, see Birn et al. (2014)).

The second (intermediate) energy range shows the parallel electron flux anisotropy. This energy range is filled by thermal $\left(E / T_{e} \in[0.1,3]\right)$ electrons that are expected to be most influenced by KAWs electric fields (Damiano et al., 2015; Artemyev et al., 2015). Moreover, this electron population is responsible for the thermal electron anisotropy in the magnetotail (Walsh et al., 2011; Artemyev et al., 2014).

The third (highest) energy range is characterised by perpendicular anisotropy that is presumably associated with betatron electron acceleration at the dipolarization front (Fu et al., 2013; Birn et al., 2012, 2013).

Note, that in the considered frequency range perpendicular electric field component usually have larger values than parallel one (both for our observations and for the theoretical predictions), so right panels on Figs. 7 and 8 cover a wider range of amplitudes than left panels do. Moreover, events with larger parallel field are rarer, i.e in the rightmost bin on the left panels we have very poor statistics.

In this study we focus on the second (thermal and subthermal) electron population. Figures 7, 8 show that the field-aligned anisotropy of this population increases with the growth of the electric field amplitude. Note we use both parallel and transverse fields: whereas the best correlation of anisotropy is expected for field-aligned fields, accuracy of this field measurements in the dipolarization configuration (when the main magnetic field component is almost along the spacecraft spin axes) is lower than accuracy of perpendicular component measurements. KAW dispersion assumes that larger perpendicular electric field component would correspond to larger field-aligned component.

The electron acceleration by KAWs is controlled by wave field-aligned electric fields $E_{\|}$, whereas typical accelerated energy is scaled with the wave scalar potential $\phi$ that can be defined as $E_{\|}=-\nabla_{\|} \phi$ (Damiano et al., 2015; Artemyev et al., 2015). Note that effective potential describes a combined contributions of electrostatic and electromagnetic fields to $E_{\|}$(Stasiewicz et al., 2000). To compare energies of field-aligned electron population with model predictions of electron acceleration by KAWs, we determine $\phi$ from $E_{\|}$spectra and KAW dispersion relation (see Eq. (3)). Panel (c) in Figs. 4 shows time series of such reconstructed $\phi$ that is used to normalize electron energy spectra. Figures 7, 8 show that energies of anisotropic electron population are well within $\sim 3|\phi|$, whereas the most anisotropic electrons have energies below $|\phi|$. This result confirms the scenario of the field-aligned electron acceleration by KAWs.

\section{Statistical analysis}

In this section we analyze of flux anisotropy and KAWs' electric fields for the entire statistics of 81 events (see Fig. 1). Fig. 9 shows averaged wave spectra and ratios of wave spectra for the entire statistics. The ratio $B_{\|} / B_{\perp} \sim 1$ for a wide frequency range, i.e. there is some contribution of compressional waves in the low frequency range $\left(\sim 10^{-2}-10^{-1} \mathrm{~Hz}\right.$ where measured compressional magnetic field fluctuations well exceed expectations from KAWs' dispersion relation, see Fig. 9 (c)). Such low frequency compressional fluctuations are quite typical for high-beta plasma sheet, especially during strong plasma flows (Bauer 


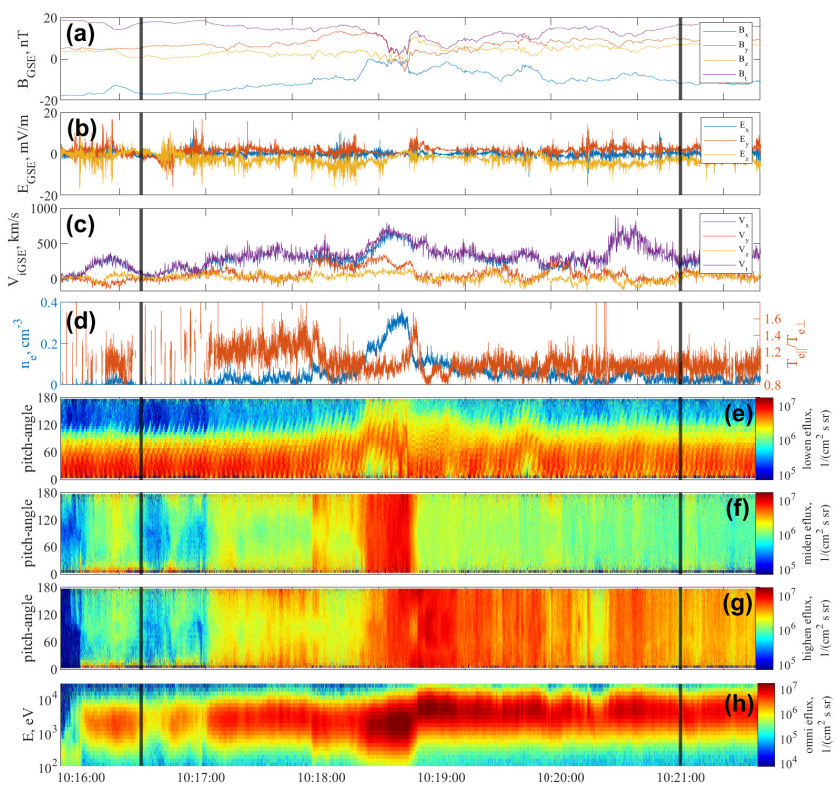

(a) First example (2017-08-18)

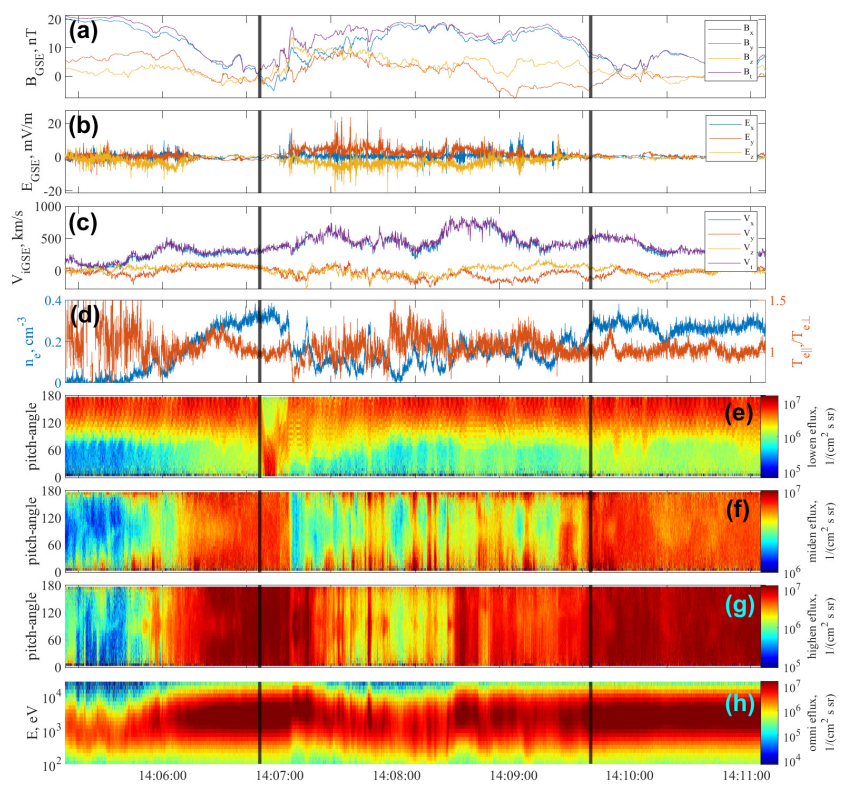

(b) Second example (2018-06-22)

Figure 3. Two intervals with wave activity sharing properties of KAWs. (a): GSE magnetic field vector; (b): GSE electric field vector; (c): GSE ion bulk velocity; (d): electron density and temperature anisotropy, (e)-(g): electron pitch-angle distributions for three energy ranges: (e) $[0,200] \mathrm{eV}$, (f) $[200,2000] \mathrm{eV}$, (g) $[2,30] \mathrm{keV}$; (h): electron energy spectrum. Vertical black lines bound time interval used for wave spectral analysis.

et al., 1995; Zelenyi et al., 2015). Besides flapping waves (Fruit et al., 2002; Volwerk et al., 2004b; Runov et al., 2005), there is a wide range of magnetic holes, localized dips of magnetic field magnitude, contributing to compressional fluctuations on the low frequency range (Ergun et al., 2020). Due to Doppler shift of all these low frequency fluctuations, these are well mixed with KAWs and we cannot distinguish KAWs contribution to $B_{\|}$in the low frequency range. However, already at frequency $>0.1 \mathrm{~Hz}$ KAWs' contribution dominates and observed ratio $B_{\|} / B_{\perp}$ follows theoretical expectations.

The effect of non-KAWs contribution to magnetic and electric field fluctuations at low frequencies is also seen in $E_{\perp} / B_{\perp}$ ratio that is $\sim 2-8 v_{A}$ instead of theoretical prediction $E_{\perp} / B_{\perp}=v_{A}$ (Fig. 9(b)). Transverse electric field fluctuations are stronger than one would expect for Alfv́en waves, and these additional fluctuation intensity likely comes from convection of compressional magnetic field structures, i.e. strong plasma flow converts almost stationary magnetic field fluctuations (low frequency flapping waves, magnetic holes) to electric field fluctuations (Volwerk et al., 2003; Zelenyi et al., 2015). For larger frequency $(>0.1 \mathrm{~Hz})$ the ratio $E_{\perp} / B_{\perp}$ follows well the prediction of KAWs dispersion, i.e. transverse electric field fluctuations grows with frequency (dominated by the Doppler shift) faster than magnetic field fluctuations (Chaston et al., 2005, 2012). Thus, Fig. 9 suggests that magnetic and electric fields (for frequency $>0.1 \mathrm{~Hz}$ ) in selected intervals are dominated by KAWs. 


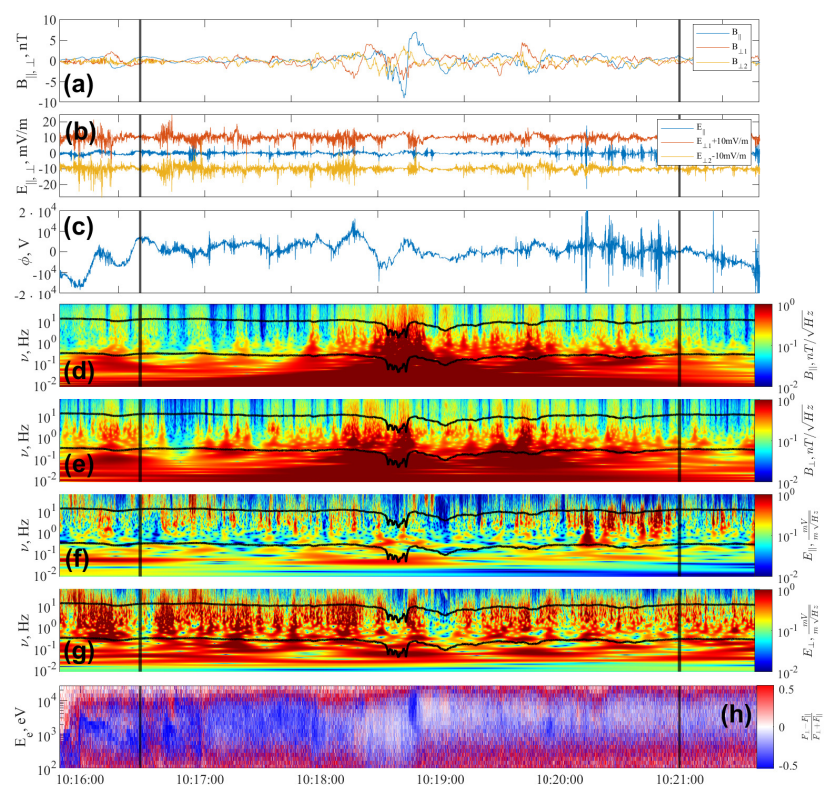

(a) First example (2017-08-18)

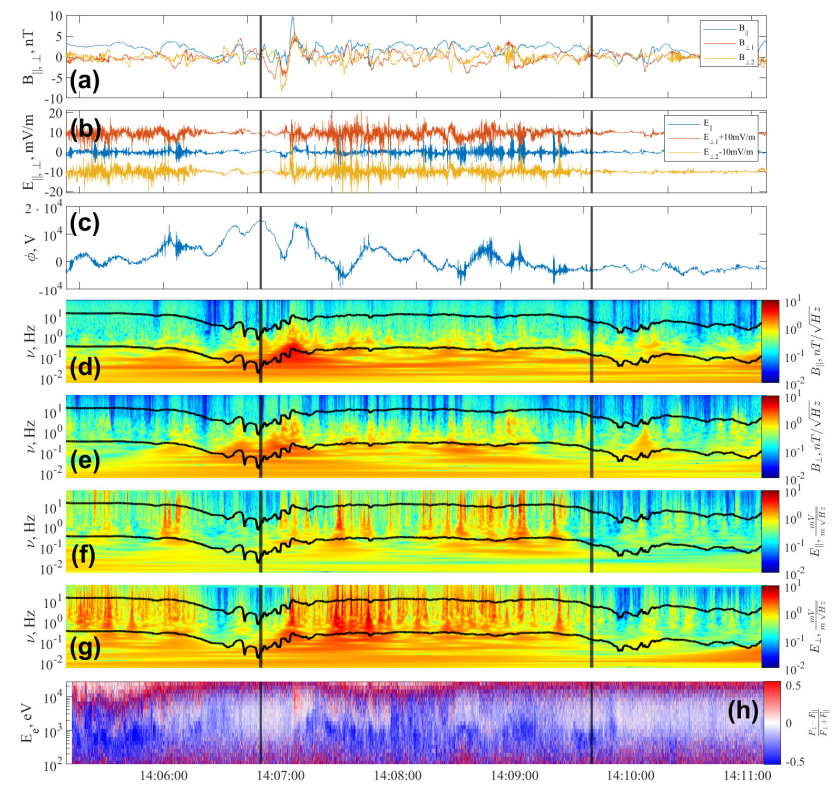

(b) Second example (2018-06-22)

Figure 4. The same intervals as on Fig. 3. (a): wave magnetic field vector in field-align coordinate system; (b): wave electric field vector in field-align coordinate system; (c): electrostatic potential estimated from the equation 3; (d)-(g): magnitudes of wavelet spectra of parallel and perpendicular wave magnetic and electric field powers; (h): electron flux anisotropy. Vertical black lines bound time interval used for wave spectral analysis. Black curves show ion cyclotron and lower hybrid frequencies.

Fig. 10 shows statistical results for electron flux anisotropy, that are quite similar to results shown for individual events in Figs. 7, 8. Energy range (normalized on electron temperature or KAWs' electrostatic potential) of anisotropic electron population increases with the increase of field-aligned and transverse electric field amplitude. This anisotropic population occupies $E / T_{e}<1$ energy range, that well coincides with the $E /|\phi|<1$ range expected from models of electron field-aligned acceleration by KAWs (Damiano et al., 2015; Damiano et al., 2016; Artemyev et al., 2015).

\section{Discussion}

We show that electron interaction with KAWs is the perspective candidate for generation of the field-aligned anisotropic electron population. This population is often observed in the Earth's magnetotail (Walsh et al., 2011; Artemyev et al., 2014), but its origins remain unestablished. Energies of field-aligned electrons can reach several keV (Sergeev et al., 2001; Yushkov et al., 2017), whereas the ionosphere outflow is expected to be limited to sub-keV energies (Walsh et al., 2013). Thus, some acceleration mechanism is needed for shaping this population. The most popular acceleration mechanism is the electron acceleration by field-aligned electric fields within the reconnection region (Asano et al., 2010; Egedal et al., 2013; Angelopoulos et al., 

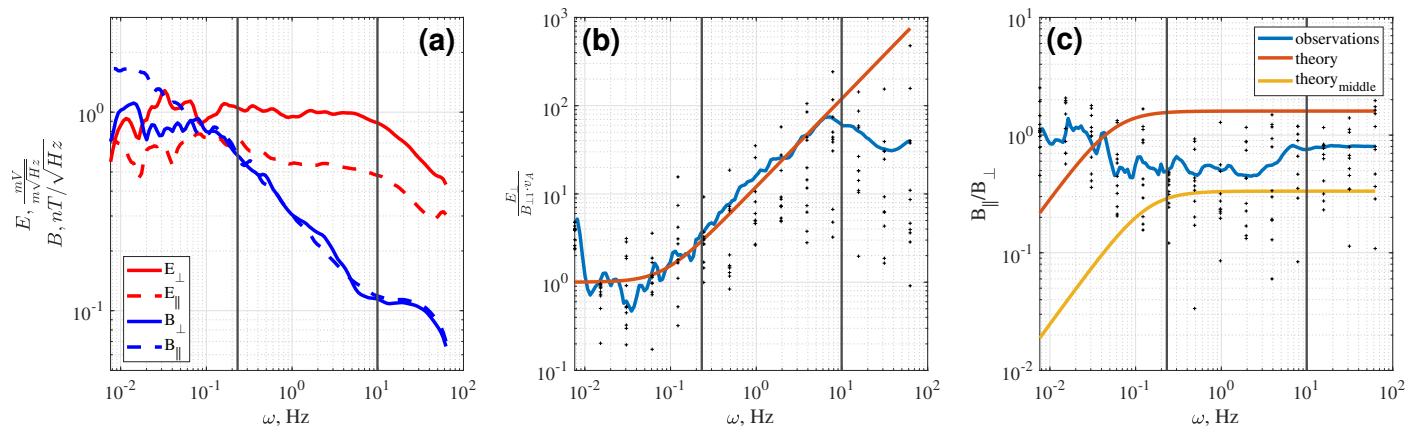

Figure 5. Theoretical and observed wave field spectra and spectra ratios for the first event (2017-08-18) averaged over 10:16:30-10:21:00. (a): averaged over selected time interval wavelet spectra of parallel/perpendicular electric and magnetic field components. (b): averaged over selected time interval ratio of perpendicular electric and magnetic field components wavelet spectra and theoretical prediction. (c): averaged over selected time interval wavelet spectra ratio of parallel and perpendicular magnetic field components and two theoretical predictions: red line shows results for observed plasma parameters and yellow line shows results for plasma parameters estimates at some distance from the equatorial plane (see text for details). Black markers show spread in time on several frequencies. Vertical lines show averaged values of ion cyclotron and lower hybrid frequencies.

2020) but observations suggest that field-aligned anisotropic electrons are not necessary seen around spatially localized reconnection region (Artemyev et al., 2020). Fast plasma flows are known as primary regions of charged particle acceleration in the magnetotail (Birn et al., 2013, 2014; Ukhorskiy et al., 2017; Gabrielse et al., 2014, 2016), but these flows are mainly associated with compressional transverse electron heating (Khotyaintsev et al., 2011; Zhang et al., 2019). Our results suggest that KAWs associated with fast plasma flows (Chaston et al., 2012) can support the mechanism of the field-aligned electron acceleration. Thus, fast flows are indeed the primary acceleration regions, but field-aligned electron acceleration is not directly resulted from electron interaction with dipolarization fronts (fast plasma flow fronts) and require KAWs' generation and further dissipation due to Landau resonance with electrons (Sharma Pyakurel et al., 2018; Gurram et al., 2020).

KAWs as a natural mode of Alfv́en waves in hot ion plasma (Stasiewicz et al., 2000) are suggested to be generated by several different mechanisms, e.g., mode conversion (Hasegawa and Chen, 1975; Hasegawa, 1976; Lin et al., 2012; Johnson and Cheng, 1997), phase mixing (Guo et al., 2015) and magnetic reconnection (Chaston et al., 2005, 2009; Liang et al., 2016; Wang et al., 2019b; Cheng et al., 2020). These waves are believed to be responsible for energy transfer from the magnetotail to 

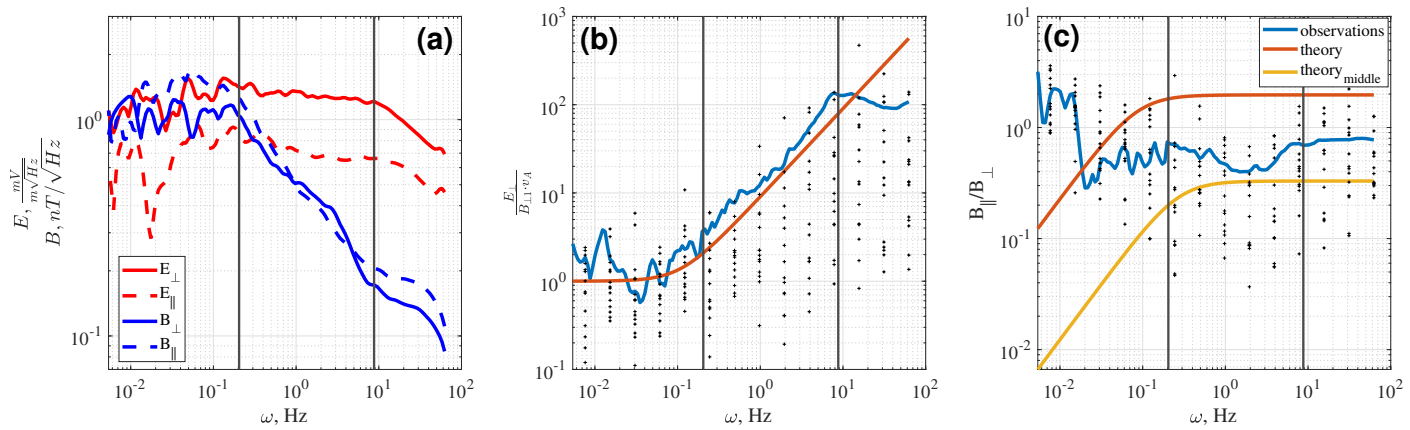

Figure 6. Same as in Fig. 5 for the second event (2018-06-22). Spectra averaged over 14:06:50-14:09:40.

the ionosphere (Lysak and Song, 2003; Keiling et al., 2003) and ionospheric electron outflow can be a response for such energy deposition (Lysak, 1990; Keiling, 2009). Thus, KAW electric field correlation with the field-aligned electron population can be, at least partially, due to the outflow mechanism. However, such outflow is expected to be north-south asymmetric (due to asymmetry of ionosphere properties Haaland et al. (e.g., 2017); Laundal et al. (e.g., 2017)) and low-energy ( $<100$ eV). Indeed, MMS FPI measurements show parallel/anti-parallel asymmetry for low energy electrons for a wider energy range up to one $\mathrm{keV}$. Presence of accelerated field-aligned anisotropic electrons and good correlation of their energy range with the upper limit of KAWs acceleration, the scalar potential amplitude $\phi$, suggests that electron acceleration by KAWs are not less important in production of the electron field-aligned anisotropy than more traditionally considered ionosphere outflow (Walsh et al., 2013).

\section{Conclusions}

In this study we have collected statistics of 81 fast plasma flow events accompanied by observations of KAWs. Case and statistical studies show a correlation between the KAWs' electric field magnitudes and thermal/subthermal electron $(\sim 1-$ $2 \mathrm{keV}$ ) flux anisotropy: wider energy range of electron anisotropic population corresponds to higher KAWs' electric field intensity. Energies of this population are well within the upper limit of electrons accelerated by KAWs, i.e. KAWs' electrostatic potential. These results indicate an important role of KAWs in production of thermal field-aligned electron population typically observed in the Earth's magnetotail. 

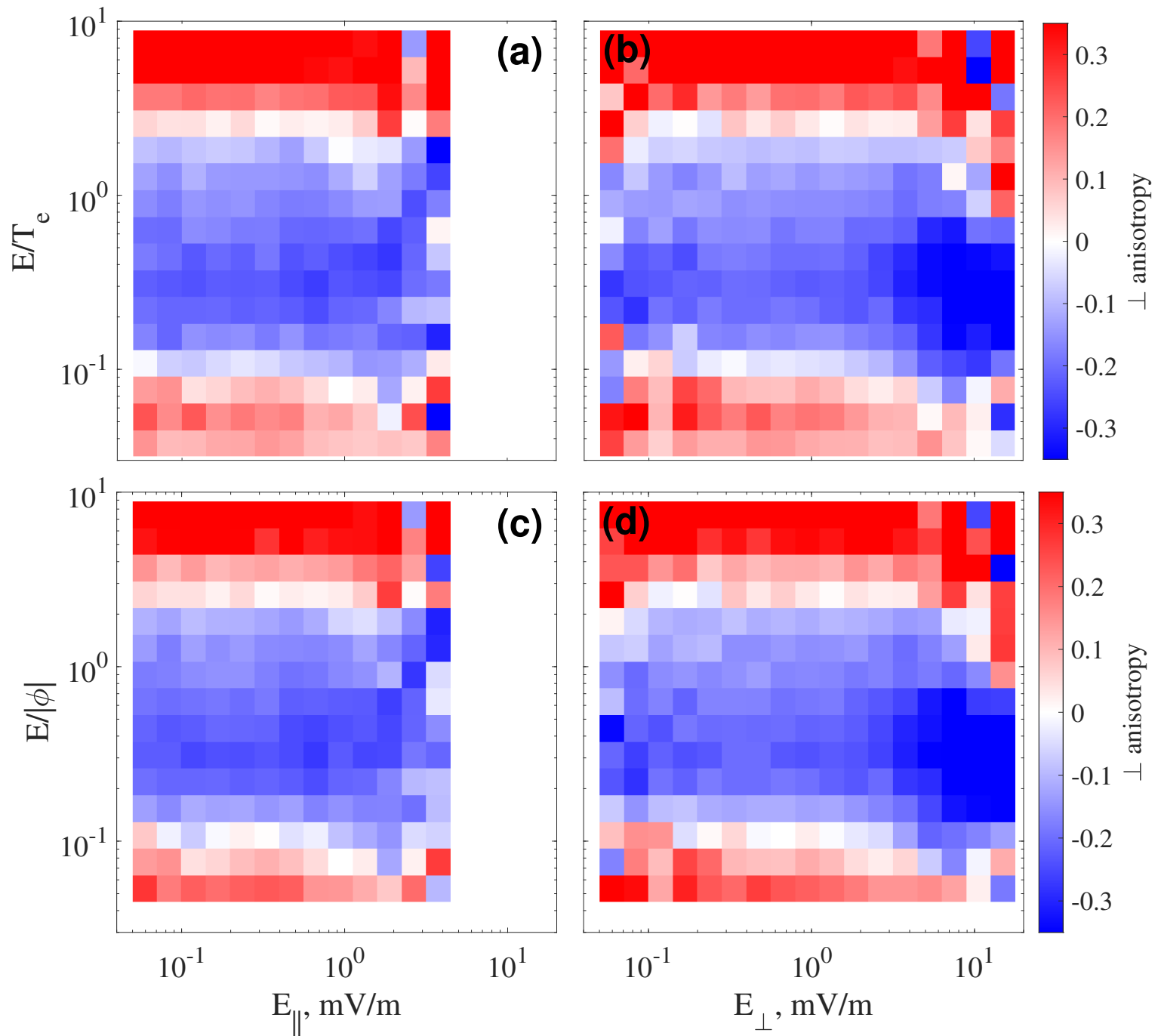

Figure 7. Electron flux anisotropy as function of parallel and perpendicular wave electric fields for the first event (2017-08-18) averaged over 10:16:30-10:21:00. (a), (b): electron energy normalized on the electron temperature; (c), (d): electron energy normalized on absolute value of electrostatic potential, estimated from the Eq. 3. 

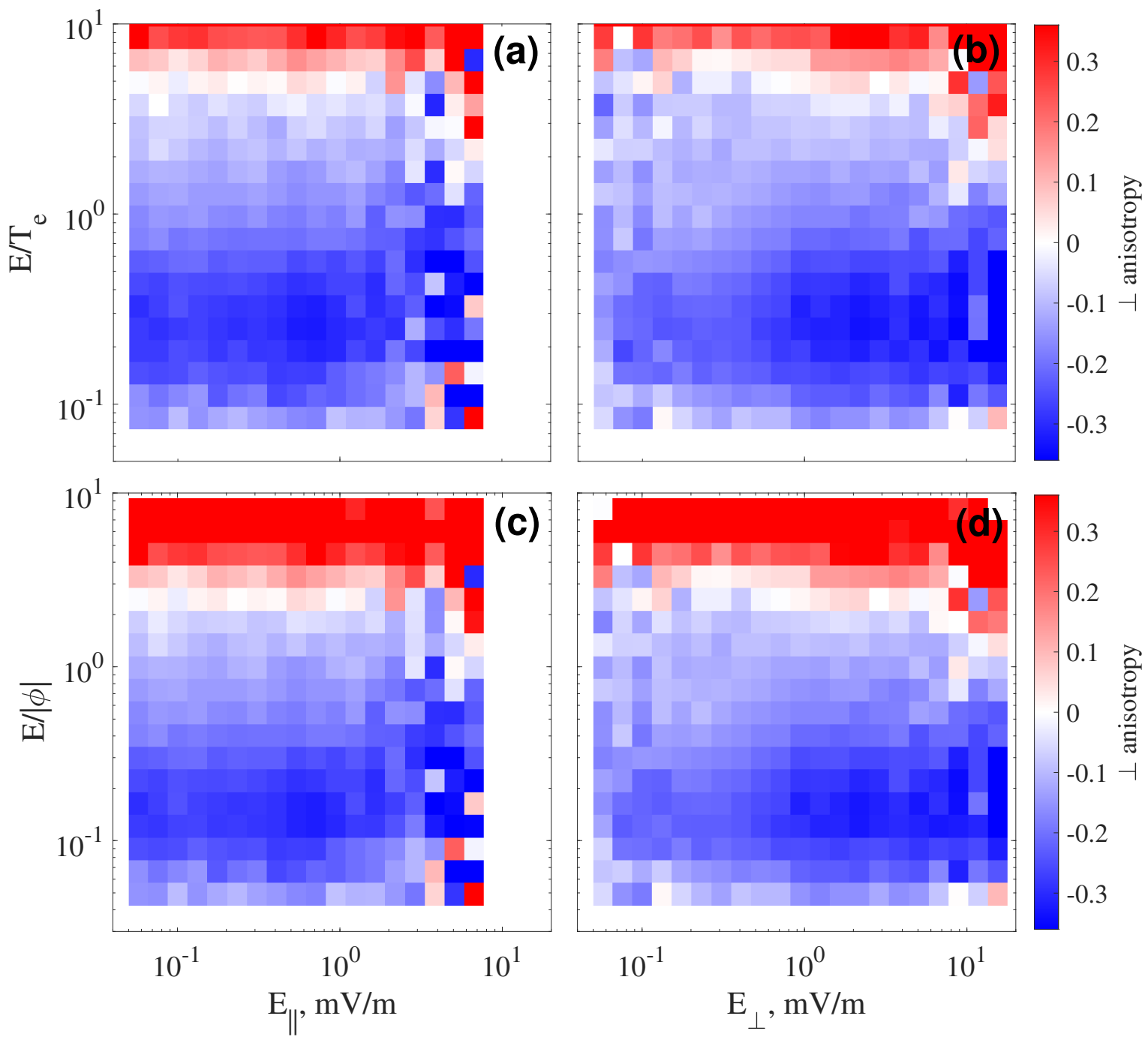

Figure 8. Same as in Fig. 7 for the second event (2018-06-22). Data averaged over 14:06:50-14:09:40. 
https://doi.org/10.5194/angeo-2020-76

Preprint. Discussion started: 27 November 2020

(c) Author(s) 2020. CC BY 4.0 License.

(c) (i)
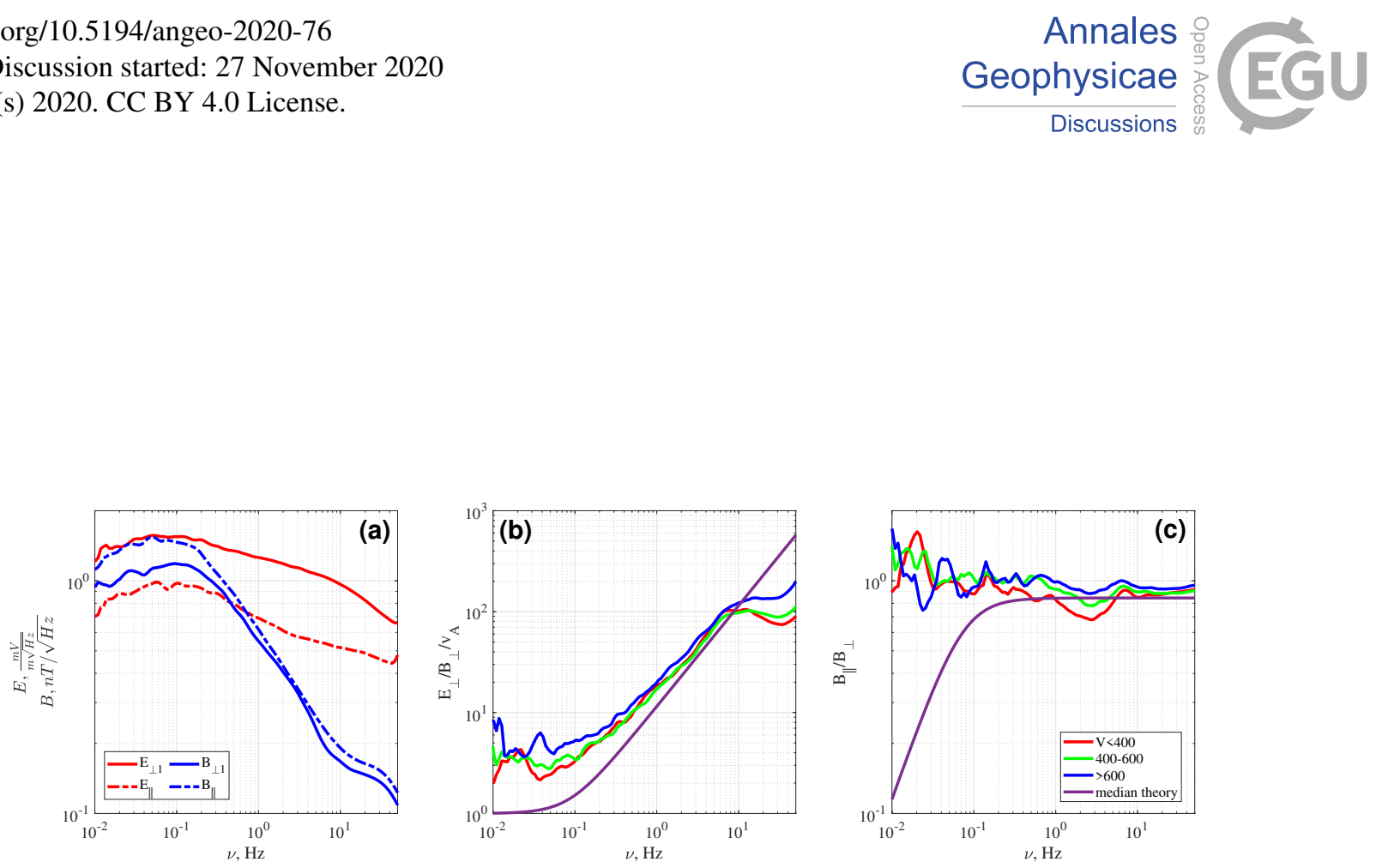

Figure 9. Averaged wave field spectra for the entire statistics. (a): averaged wavelet spectra of parallel and perpendicular electric and magnetic field components. (b): averaged ratios of spectra of perpendicular electric and magnetic field components and theoretical estimates. (c): averaged ratios of spectra of parallel and perpendicular magnetic field components and theoretical estimates.

255 Acknowledgements. Work of L.A.S, A.A.V., P.A.A. was supported by Russian Scientific Foundation (project no. 19-12-00313). Work of E.V.P. was supported by the Austrian Science Fund (FWF): I 3506-N27. Data access and processing was done using SPEDAS V3.1, see Angelopoulos19. MMS (FGM, FPI, SDP/ADP) data were downloaded from databases https://lasp.colorado.edu/mms/sdc/public/. 

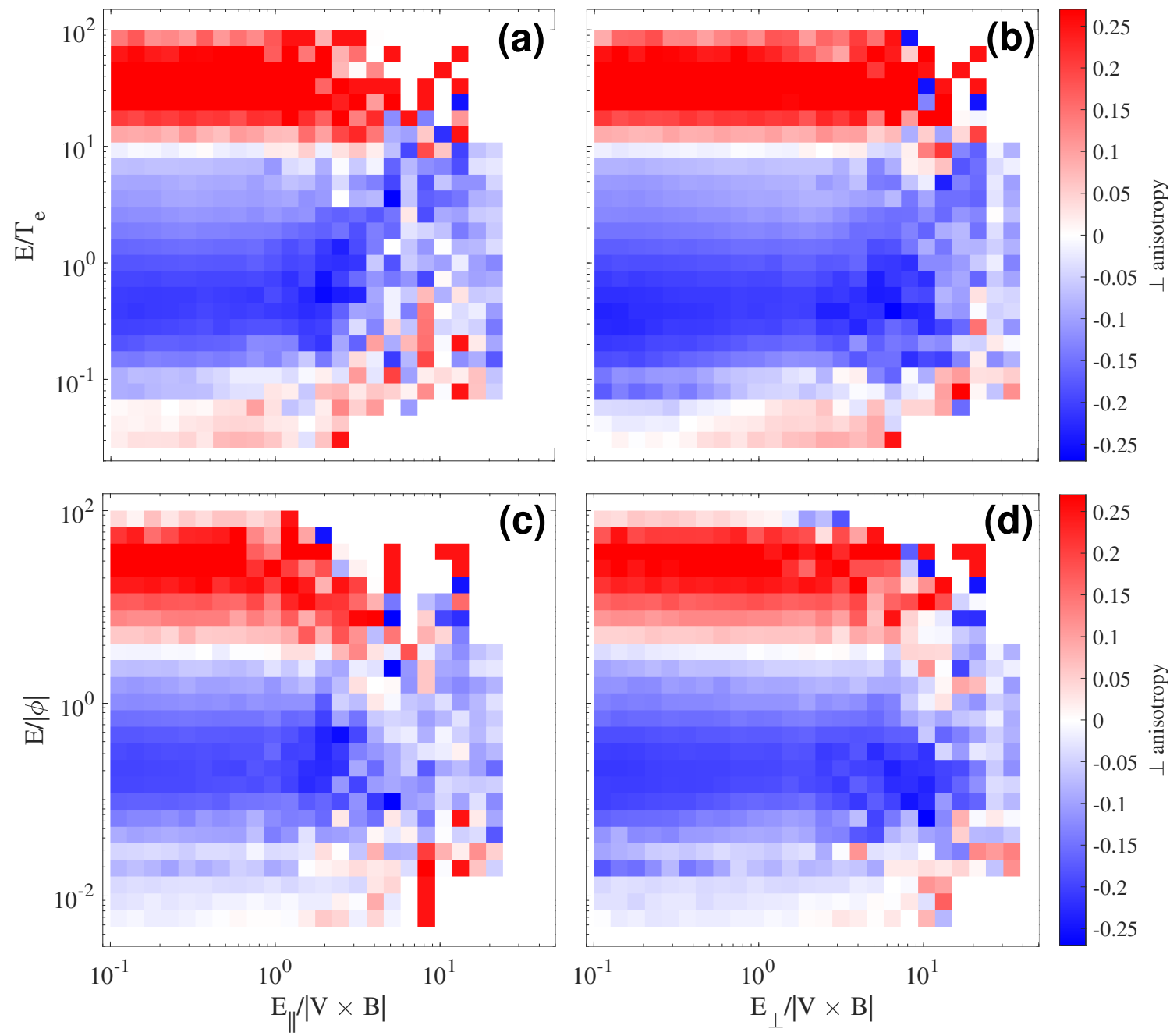

Figure 10. Statistical study of electron flux anisotropy. Figure format repeats the one shown in Fig. 7, but here we use dataset averaged over the entire statistics of 81 events. 
https://doi.org/10.5194/angeo-2020-76

Preprint. Discussion started: 27 November 2020

(c) Author(s) 2020. CC BY 4.0 License.

(c) (i)

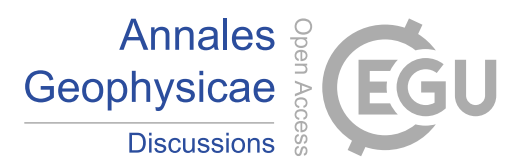

\section{References}

Angelopoulos, V.: The THEMIS Mission, Space Sci. Rev. , 141, 5-34, https://doi.org/10.1007/s11214-008-9336-1, 2008.

Angelopoulos, V., Baumjohann, W., Kennel, C. F., Coronti, F. V., Kivelson, M. G., Pellat, R., Walker, R. J., Luehr, H., and Paschmann, G.: Bursty bulk flows in the inner central plasma sheet, J. Geophys. Res., 97, 4027-4039, https://doi.org/10.1029/91JA02701, 1992.

Angelopoulos, V., Cruce, P., Drozdov, A., Grimes, E. W., Hatzigeorgiu, N., King, D. A., Larson, D., Lewis, J. W., McTiernan, J. M., Roberts, D. A., Russell, C. L., Hori, T., Kasahara, Y., Kumamoto, A., Matsuoka, A., Miyashita, Y., Miyoshi, Y., Shinohara, I., Teramoto, M., Faden, J. B., Halford, A. J., McCarthy, M., Millan, R. M., Sample, J. G., Smith, D. M., Woodger, L. A., Masson, A., Narock, A. A., Asamura, K., Chang, T. F., Chiang, C.-Y., Kazama, Y., Keika, K., Matsuda, S., Segawa, T., Seki, K., Shoji, M., Tam, S. W. Y., Umemura, N., Wang, B.-J., Wang, S.-Y., Redmon, R., Rodriguez, J. V., Singer, H. J., Vandegriff, J., Abe, S., Nose, M., Shinbori, A., Tanaka, Y.-M., UeNo, S., Andersson, L., Dunn, P., Fowler, C., Halekas, J. S., Hara, T., Harada, Y., Lee, C. O., Lillis, R., Mitchell, D. L., Argall, M. R., Bromund, K., Burch, J. L., Cohen, I. J., Galloy, M., Giles, B., Jaynes, A. N., Le Contel, O., Oka, M., Phan, T. D., Walsh, B. M., Westlake, J., Wilder, F. D., Bale, S. D., Livi, R., Pulupa, M., Whittlesey, P., DeWolfe, A., Harter, B., Lucas, E., Auster, U., Bonnell, J. W., Cully, C. M., Donovan, E., Ergun, R. E., Frey, H. U., Jackel, B., Keiling, A., Korth, H., McFadden, J. P., Nishimura, Y., Plaschke, F., Robert, P., Turner, D. L., Weygand, J. M., Candey, R. M., Johnson, R. C., Kovalick, T., Liu, M. H., McGuire, R. E., Breneman, A., Kersten, K., and Schroeder, P.: The Space Physics Environment Data Analysis System (SPEDAS), Space Sci. Rev. , 215, 9, https://doi.org/10.1007/s11214-018-0576-4, 2019.

Angelopoulos, V., Artemyev, A., Phan, T. D., and Miyashita, Y.: Near-Earth magnetotail reconnection powers space storms, Nature Physics, 16, 317-321, https://doi.org/10.1038/s41567-019-0749-4, 2020.

Artemyev, A. V., Petrukovich, A. A., Nakamura, R., and Zelenyi, L. M.: Cluster statistics of thin current sheets in the Earth magnetotail: specifics of the dawn flank, proton temperature profiles and electrostatic effects., J. Geophys. Res., 116, A0923, https://doi.org/10.1029/2011JA016801, 2011a.

Artemyev, A. V., Zelenyi, L. M., Petrukovich, A. A., and Nakamura, R.: Hot electrons as tracers of large-scale structure of magnetotail current sheets, Geophys. Res. Lett., 38, L14 102, https://doi.org/10.1029/2011GL047979, 2011 b.

Artemyev, A. V., Walsh, A. P., Petrukovich, A. A., Baumjohann, W., Nakamura, R., and Fazakerley, A. N.: Electron pitch angle/energy distribution in the magnetotail, J. Geophys. Res., 119, 7214-7227, https://doi.org/10.1002/2014JA020350, 2014.

Artemyev, A. V., Rankin, R., and Blanco, M.: Electron trapping and acceleration by kinetic Alfven waves in the inner magnetosphere, J. Geophys. Res., 120, 10, https://doi.org/10.1002/2015JA021781, 2015.

Artemyev, A. V., Vasko, I. Y., Angelopoulos, V., and Runov, A.: Effects of electron pressure anisotropy on current sheet configuration, Physics of Plasmas, 23, 092901, https://doi.org/10.1063/1.4961926, 2016.

Artemyev, A. V., Angelopoulos, V., Liu, J., and Runov, A.: Electron currents supporting the near-Earth magnetotail during current sheet thinning, Geophys. Res. Lett., 44, 5-11, https://doi.org/10.1002/2016GL072011, 2017.

Artemyev, A. V., Angelopoulos, V., Vasko, I. Y., Petrukovich, A. A., Runov, A., Saito, Y., Avanov, L. A., Giles, B. L., Russell, C. T., and Strangeway, R. J.: Contribution of Anisotropic Electron Current to the Magnetotail Current Sheet as a Function of Location and Plasma Conditions, Journal of Geophysical Research: Space Physics, 125, e2019JA027 251, https://doi.org/10.1029/2019JA027251, https: //agupubs.onlinelibrary.wiley.com/doi/abs/10.1029/2019JA027251, e2019JA027251 10.1029/2019JA027251, 2020. 
https://doi.org/10.5194/angeo-2020-76

Preprint. Discussion started: 27 November 2020

(c) Author(s) 2020. CC BY 4.0 License.

(c) (i)

Asano, Y., Shinohara, I., Retinò, A., Daly, P. W., Kronberg, E. A., Takada, T., Nakamura, R., Khotyaintsev, Y. V., Vaivads, A., Nagai, T., Baumjohann, W., Fazakerley, A. N., Owen, C. J., Miyashita, Y., Lucek, E. A., and Rème, H.: Electron acceleration signatures in the magnetotail associated with substorms, J. Geophys. Res., 115, A05 215, https://doi.org/10.1029/2009JA014587, 2010.

Bashir, M. F., Chen, L., and Ilie, R.: Theoretical Prediction of Asymmetric Instability of Drift Kinetic Alfven Waves in Anisotropic Plasmas, Journal of Geophysical Research (Space Physics), 124, 4414-4423, https://doi.org/10.1029/2019JA026615, 2019.

Bauer, T. M., Baumjohann, W., Treumann, R. A., Sckopke, N., and Lühr, H.: Low-frequency waves in the near-Earth plasma sheet, J. Geophys. Res., 100, 9605-9618, https://doi.org/10.1029/95JA00136, 1995.

Baumjohann, W., Paschmann, G., and Luehr, H.: Characteristics of high-speed ion flows in the plasma sheet, J. Geophys. Res., 95, 38013809, https://doi.org/10.1029/JA095iA04p03801, 1990.

Birn, J., Artemyev, A. V., Baker, D. N., Echim, M., Hoshino, M., and Zelenyi, L. M.: Particle acceleration in the magnetotail and aurora, Space Sci. Rev. , 173, 49-102, https://doi.org/10.1007/s11214-012-9874-4, 2012.

Birn, J., Hesse, M., Nakamura, R., and Zaharia, S.: Particle acceleration in dipolarization events, J. Geophys. Res., 118, 1960-1971, https://doi.org/10.1002/jgra.50132, 2013.

Birn, J., Runov, A., and Hesse, M.: Energetic electrons in dipolarization events: Spatial properties and anisotropy, Journal of Geophysical Research (Space Physics), 119, 3604-3616, https://doi.org/10.1002/2013JA019738, 2014.

Breuillard, H., Le Contel, O., Retino, A., Chasapis, A., Chust, T., Mirioni, L., Graham, D. B., Wilder, F. D., Cohen, I., Vaivads, A., Khotyaintsev, Y. V., Lindqvist, P.-A., Marklund, G. T., Burch, J. L., Torbert, R. B., Ergun, R. E., Goodrich, K. A., Macri, J., Needell, J., Chutter, M., Rau, D., Dors, I., Russell, C. T., Magnes, W., Strangeway, R. J., Bromund, K. R., Plaschke, F., Fischer, D., Leinweber, H. K., Anderson, B. J., Le, G., Slavin, J. A., Kepko, E. L., Baumjohann, W., Mauk, B., Fuselier, S. A., and Nakamura, R.: Multispacecraft analysis of dipolarization fronts and associated whistler wave emissions using MMS data, Geophys. Res. Lett., 43, 7279-7286, https://doi.org/10.1002/2016GL069188, 2016.

Burch, J. L., Moore, T. E., Torbert, R. B., and Giles, B. L.: Magnetospheric Multiscale Overview and Science Objectives, Space Sci. Rev. , 199, 5-21, https://doi.org/10.1007/s11214-015-0164-9, 2016.

Chaston, C. C., Peticolas, L. M., Carlson, C. W., McFadden, J. P., Mozer, F., Wilber, M., Parks, G. K., Hull, A., Ergun, R. E., Strangeway, R. J., Andre, M., Khotyaintsev, Y., Goldstein, M. L., AcuñA, M., Lund, E. J., Reme, H., Dandouras, I., Fazakerley, A. N., and Balogh, A.: Energy deposition by Alfvén waves into the dayside auroral oval: Cluster and FAST observations, J. Geophys. Res., 110, A02211, https://doi.org/10.1029/2004JA010483, 2005.

Chaston, C. C., Salem, C., Bonnell, J. W., Carlson, C. W., Ergun, R. E., Strangeway, R. J., and McFadden, J. P.: The Turbulent Alfvénic Aurora, Physical Review Letters, 100, 175003, https://doi.org/10.1103/PhysRevLett.100.175003, 2008.

Chaston, C. C., Johnson, J. R., Wilber, M., Acuna, M., Goldstein, M. L., and Reme, H.: Kinetic Alfvén Wave Turbulence and Transport through a Reconnection Diffusion Region, Physical Review Letters, 102, 015001, https://doi.org/10.1103/PhysRevLett.102.015001, 2009.

Chaston, C. C., Bonnell, J. W., Clausen, L., and Angelopoulos, V.: Energy transport by kinetic-scale electromagnetic waves in fast plasma sheet flows, J. Geophys. Res., 117, A09202, https://doi.org/10.1029/2012JA017863, 2012.

Chaston, C. C., Yao, Y., Lin, N., Salem, C., and Ueno, G.: Ion heating by broadband electromagnetic waves in the magnetosheath and across the magnetopause, J. Geophys. Res., 118, 5579-5591, https://doi.org/10.1002/jgra.50506, 2013.

Chaston, C. C., Bonnell, J. W., Wygant, J. R., Mozer, F., Bale, S. D., Kersten, K., Breneman, A. W., Kletzing, C. A., Kurth, W. S., Hospodarsky, G. B., Smith, C. W., and MacDonald, E. A.: Observations of kinetic scale field line resonances, Geophys. Res. Lett., 41, 209-215, https://doi.org/10.1002/2013GL058507, 2014. 
https://doi.org/10.5194/angeo-2020-76

Preprint. Discussion started: 27 November 2020

(c) Author(s) 2020. CC BY 4.0 License.

(c) (i)

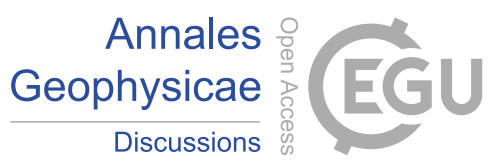

Cheng, L., Lin, Y., Perez, J., Johnson, J. R., and Wang, X.: Kinetic Alfvén Waves From Magnetotail to the Ionosphere in Global Hybrid Simulation Associated With Fast Flows, Journal of Geophysical Research: Space Physics, 125, e2019JA027 062, 2020.

Damiano, P. A., Johnson, J. R., and Chaston, C. C.: Ion temperature effects on magnetotail Alfvén wave propagation and electron energization, J. Geophys. Res., 120, 5623-5632, https://doi.org/10.1002/2015JA021074, 2015.

Damiano, P. A., Johnson, J. R., and Chaston, C. C.: Ion gyroradius effects on particle trapping in kinetic Alfvn waves along auroral field lines, J. Geophys. Res., 121, 10,831-10,844, https://doi.org/10.1002/2016JA022566, http://dx.doi.org/10.1002/2016JA022566, 2016.

Deng, X., Ashour-Abdalla, M., Zhou, M., Walker, R., El-Alaoui, M., Angelopoulos, V., Ergun, R. E., and Schriver, D.: Wave and particle characteristics of earthward electron injections associated with dipolarization fronts, J. Geophys. Res., 115, A09225, https://doi.org/10.1029/2009JA015107, 2010.

Egedal, J., Le, A., and Daughton, W.: A review of pressure anisotropy caused by electron trapping in collisionless plasma, and its implications for magnetic reconnection, Physics of Plasmas, 20, 061 201, https://doi.org/10.1063/1.4811092, 2013.

Ergun, R. E., Goodrich, K. A., Stawarz, J. E., Andersson, L., and Angelopoulos, V.: Large-amplitude electric fields associated with bursty bulk flow braking in the Earth's plasma sheet, J. Geophys. Res., 120, 1832-1844, https://doi.org/10.1002/2014JA020165, 2015.

Ergun, R. E., Tucker, S., Westfall, J., Goodrich, K. A., Malaspina, D. M., Summers, D., Wallace, J., Karlsson, M., Mack, J., Brennan, N., Pyke, B., Withnell, P., Torbert, R., Macri, J., Rau, D., Dors, I., Needell, J., Lindqvist, P.-A., Olsson, G., and Cully, C. M.: The Axial Double Probe and Fields Signal Processing for the MMS Mission, Space Sci. Rev. , 199, 167-188, https://doi.org/10.1007/s11214-014-0115-x, 2016.

Ergun, R. E., Ahmadi, N., Kromyda, L., Schwartz, S. J., Chasapis, A., Hoilijoki, S., Wilder, F. D., Stawarz, J. E., Goodrich, K. A., Turner, D. L., Cohen, I. J., Bingham, S. T., Holmes, J. C., Nakamura, R., Pucci, F., Torbert, R. B., Burch, J. L., Lindqvist, P. A., Strangeway, R. J., Le Contel, O., and Giles, B. L.: Observations of Particle Acceleration in Magnetic Reconnection-driven Turbulence, Astrophys. J., 898, 154, https://doi.org/10.3847/1538-4357/ab9ab6, 2020.

Escoubet, C. P., Fehringer, M., and Goldstein, M.: Introduction: The Cluster mission, Annales Geophysicae, 19, 1197-1200, https://doi.org/10.5194/angeo-19-1197-2001, 2001.

Fruit, G., Louarn, P., Tur, A., and Le Quéau, D.: On the propagation of magnetohydrodynamic perturbations in a Harris-type current sheet 1. Propagation on discrete modes and signal reconstruction, J. Geophys. Res., 107, 1411-+, https://doi.org/10.1029/2001JA009212, 2002.

Fu, H. S., Khotyaintsev, Y. V., Vaivads, A., André, M., and Huang, S. Y.: Occurrence rate of earthward-propagating dipolarization fronts, Geophys. Res. Lett., 39, L10101, https://doi.org/10.1029/2012GL051784, 2012.

Fu, H. S., Khotyaintsev, Y. V., Vaivads, A., Retinò, A., and André, M.: Energetic electron acceleration by unsteady magnetic reconnection, Nature Physics, 9, 426-430, https://doi.org/10.1038/nphys2664, 2013.

Gabrielse, C., Angelopoulos, V., Runov, A., and Turner, D. L.: Statistical characteristics of particle injections throughout the equatorial magnetotail, J. Geophys. Res., 119, 2512-2535, https://doi.org/10.1002/2013JA019638, 2014.

Gabrielse, C., Harris, C., Angelopoulos, V., Artemyev, A., and Runov, A.: The role of localized inductive electric fields in electron injections around dipolarizing flux bundles, J. Geophys. Res., 121, 9560-9585, https://doi.org/10.1002/2016JA023061, 2016.

Goldman, M. V., Oppenheim, M. M., and Newman, D. L.: Theory of localized bipolar wave-structures and nonthermal particle distributions in the auroral ionosphere, Nonlinear Processes in Geophysics, 6, 221-228, https://doi.org/10.5194/npg-6-221-1999, 1999.

Grigorenko, E., Malykhin, A. Y., Shklyar, D., Fadanelli, S., Lavraud, B., Panov, E., Avanov, L., Giles, B., and Le Contel, O.: Investigation of Electron Distribution Functions Associated With Whistler Waves at Dipolarization Fronts in the Earth's Magnetotail: MMS Observations, Journal of Geophysical Research: Space Physics, 125, e2020JA028 268, https://doi.org/10.1029/2020JA028268, 2020. 
https://doi.org/10.5194/angeo-2020-76

Preprint. Discussion started: 27 November 2020

(c) Author(s) 2020. CC BY 4.0 License.

(c) (i)

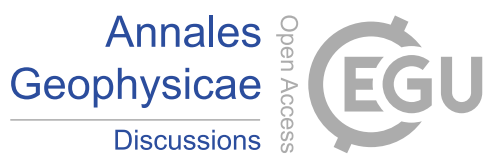

Guo, Z., Hong, M., Lin, Y., Du, A., Wang, X., Wu, M., and Lu, Q.: Generation of kinetic Alfven waves in the high-latitude near-Earth magnetotail: A global hybrid simulation, Physics of Plasmas, 22, 022 117, 2015.

Gurram, H., Egedal, J., and Daughton, W.: Mode Converting Alfvén Waves from Magnetic Reconnection Enhancing the Energy Source for the Aurora Borealis, arXiv e-prints, arXiv:2004.11755, 2020.

Haaland, S., Lybekk, B., Maes, L., Laundal, K., Pedersen, A., Tenfjord, P., Ohma, A., Østgaard, N., Reistad, J., and Snekvik, K.: North-south asymmetries in cold plasma density in the magnetotail lobes: Cluster observations, Journal of Geophysical Research: Space Physics, 122 , 136-149, 2017.

Hada, T., Nishida, A., Terasawa, T., and Hones, Jr., E. W.: Bi-directional electron pitch angle anisotropy in the plasma sheet, J. Geophys. Res., 86, 11 211-11 224, https://doi.org/10.1029/JA086iA13p11211, 1981.

Hasegawa, A.: Particle acceleration by MHD surface wave and formation of aurora, J. Geophys. Res., 81, 5083-5090, https://doi.org/10.1029/JA081i028p05083, 1976.

Hasegawa, A. and Chen, L.: Kinetic process of plasma heating due to Alfvén wave excitation, Physical Review Letters, 35, 370-373, https://doi.org/10.1103/PhysRevLett.35.370, 1975.

Hau, L.-N.: Anisotropic magnetotail equilibrium and convection, Geophys. Res. Lett., 20, 555-558, https://doi.org/10.1029/93GL00243, 1993.

Huang, H., Yu, Y., Dai, L., and Wang, T.: Kinetic Alfvén Waves Excited in Two-Dimensional Magnetic Reconnection, Journal of Geophysical Research (Space Physics), 123, 6655-6669, https://doi.org/10.1029/2017JA025071, 2018.

Huang, S., Fu, H., Yuan, Z., Vaivads, A., Khotyaintsev, Y. V., Retino, A., Zhou, M., Graham, D. B., Fujimoto, K., Sahraoui, F., et al.: Two types of whistler waves in the hall reconnection region, Journal of Geophysical Research: Space Physics, 121, 6639-6646, 2016.

Johnson, J. R. and Cheng, C. Z.: Global structure of mirror modes in the magnetosheath, J. Geophys. Res., 102, 7179-7190, https://doi.org/10.1029/96JA03949, 1997.

Kamaletdinov, S., Yushkov, E., Artemyev, A., Lukin, A., and Vasko, I.: Superthin current sheets supported by anisotropic electrons, Physics of Plasmas, 27, 082 904, 2020.

Karimabadi, H., Akimoto, K., Omidi, N., and Menyuk, C. R.: Particle acceleration by a wave in a strong magnetic field - Regular and stochastic motion, Physics of Fluids B, 2, 606-628, https://doi.org/10.1063/1.859296, 1990.

Keiling, A.: Alfvén Waves and Their Roles in the Dynamics of the Earth's Magnetotail: A Review, Space Sci. Rev. , 142, 73-156, https://doi.org/10.1007/s11214-008-9463-8, 2009.

Keiling, A., Wygant, J. R., Cattell, C. A., Mozer, F. S., and Russell, C. T.: The Global Morphology of Wave Poynting Flux: Powering the Aurora, Science, 299, 383-386, https://doi.org/10.1126/science.1080073, 2003.

Khazanov, G. V., Glocer, A., and Himwich, E. W.: Magnetosphere-ionosphere energy interchange in the electron diffuse aurora, Journal of Geophysical Research (Space Physics), 119, 171-184, https://doi.org/10.1002/2013JA019325, 2014.

Khotyaintsev, Y. V., Cully, C. M., Vaivads, A., André, M., and Owen, C. J.: Plasma Jet Braking: Energy Dissipation and Nonadiabatic Electrons, Physical Review Letters, 106, 165 001, https://doi.org/10.1103/PhysRevLett.106.165001, 2011.

Kletzing, C. A.: Electron acceleration by kinetic Alfvén waves, J. Geophys. Res., 99, 11 095-11 104, https://doi.org/10.1029/94JA00345, 1994.

Laundal, K. M., Cnossen, I., Milan, S. E., Haaland, S., Coxon, J., Pedatella, N., Förster, M., and Reistad, J. P.: North-south asymmetries in earth's magnetic field, Space Science Reviews, 206, 225-257, 2017. 
https://doi.org/10.5194/angeo-2020-76

Preprint. Discussion started: 27 November 2020

(c) Author(s) 2020. CC BY 4.0 License.

(c) (i)

Le Contel, O., Roux, A., Jacquey, C., Robert, P., Berthomier, M., Chust, T., Grison, B., Angelopoulos, V., Sibeck, D., Chaston, C. C., Cully, C. M., Ergun, B., Glassmeier, K.-H., Auster, U., McFadden, J., Carlson, C., Larson, D., Bonnell, J. W., Mende, S., Russell, C. T., Donovan, E., Mann, I., and Singer, H.: Quasi-parallel whistler mode waves observed by THEMIS during near-earth dipolarizations, Annales Geophysicae, 27, 2259-2275, https://doi.org/10.5194/angeo-27-2259-2009, 2009.

410 Liang, J., Lin, Y., Johnson, J. R., Wang, X., and Wang, Z.-X.: Kinetic Alfvén waves in three-dimensional magnetic reconnection, J. Geophys. Res., 121, 6526-6548, https://doi.org/10.1002/2016JA022505, 2016.

Lin, Y., Johnson, J. R., and Wang, X.: Three-dimensional mode conversion associated with kinetic Alfvén waves, Physical review letters, 109, $125003,2012$.

Lin, Y., Wing, S., Johnson, J. R., Wang, X. Y., Perez, J. D., and Cheng, L.: Formation and transport of entropy structures in the magnetotail simulated with a 3-D global hybrid code, Geophys. Res. Lett., 44, 5892-5899, https://doi.org/10.1002/2017GL073957, 2017.

Lindqvist, P.-A., Olsson, G., Torbert, R. B., King, B., Granoff, M., Rau, D., Needell, G., Turco, S., Dors, I., Beckman, P., Macri, J., Frost, C., Salwen, J., Eriksson, A., Åhlén, L., Khotyaintsev, Y. V., Porter, J., Lappalainen, K., Ergun, R. E., Wermeer, W., and Tucker, S.: The SpinPlane Double Probe Electric Field Instrument for MMS, Space Sci. Rev. , 199, 137-165, https://doi.org/10.1007/s11214-014-0116-9, 2016.

Lotekar, A., Vasko, I. Y., Mozer, F. S., Hutchinson, I., Artemyev, A. V., Bale, S. D., Bonnell, J. W., Ergun, R., Giles, B., Khotyaintsev, Y. V., Lindqvist, P.-A., Russell, C. T., and Strangeway, R.: Multisatellite MMS Analysis of Electron Holes in the Earth's Magnetotail: Origin, Properties, Velocity Gap, and Transverse Instability, Journal of Geophysical Research: Space Physics, 125, e2020JA028 066, https://doi.org/10.1029/2020JA028066, e2020JA028066 10.1029/2020JA028066, 2020.

Lyons, L. R.: Electron energization in the geomagnetic tail current sheet, J. Geophys. Res., 89, 5479-5487, https://doi.org/10.1029/JA089iA07p05479, 1984.

Lysak, R. L.: Electrodynamic coupling of the magnetosphere and ionosphere, Space Sci. Rev. , 52, 33-87, https://doi.org/10.1007/BF00704239, 1990.

Lysak, R. L. and Song, Y.: Kinetic theory of the Alfvén wave acceleration of auroral electrons, J. Geophys. Res., 108, 8005, https://doi.org/10.1029/2002JA009406, 2003.

Malaspina, D. M., Ukhorskiy, A., Chu, X., and Wygant, J.: A Census of Plasma Waves and Structures Associated With an Injection Front in the Inner Magnetosphere, J. Geophys. Res., 123, 2566-2587, https://doi.org/10.1002/2017JA025005, 2018.

Matsumoto, H., Kojima, H., Miyatake, T., Omura, Y., Okada, M., Nagano, I., and Tsutsui, M.: Electrotastic Solitary Waves (ESW) in the magnetotail: BEN wave forms observed by GEOTAIL, Geophys. Res. Lett., 21, 2915-2918, https://doi.org/10.1029/94GL01284, 1994.

Mingalev, O. V., Malova, H. V., Mingalev, I. V., Mel'nik, M. N., Setsko, P. V., and Zelenyi, L. M.: Model of a Thin Current Sheet in the Earth's Magnetotail with a Kinetic Description of Magnetized Electrons, Plasma Physics Reports, 44, 899-919, https://doi.org/10.1134/S1063780X18100082, https://doi.org/10.1134/S1063780X18100082, 2018.

Mozer, F. S., Agapitov, O., Artemyev, A., Drake, J. F., Krasnoselskikh, V., Lejosne, S., and Vasko, I.: Time domain structures: What and where they are, what they do, and how they are made, Geophys. Res. Lett., 42, 3627-3638, https://doi.org/10.1002/2015GL063946, 2015.

Nakamura, R., Baumjohann, W., Schödel, R., Brittnacher, M., Sergeev, V., Kubyshkina, M., Mukai, T., and Liou, K.: Earthward flow bursts, auroral streamers, and small expansions, Journal of Geophysical Research: Space Physics, 106, 10 791-10 802, 2001.

Omura, Y., Matsumoto, H., Miyake, T., and Kojima, H.: Electron beam instabilities as generation mechanism of electrostatic solitary waves in the magnetotail, J. Geophys. Res., 101, 2685-2698, https://doi.org/10.1029/95JA03145, 1996. 
https://doi.org/10.5194/angeo-2020-76

Preprint. Discussion started: 27 November 2020

(c) Author(s) 2020. CC BY 4.0 License.

(c) (i)

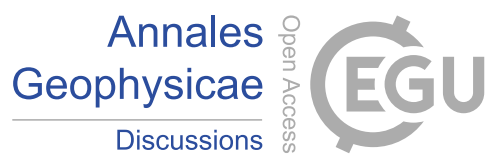

Palmadesso, P. J.: Resonance, Particle Trapping, and Landau Damping in Finite Amplitude Obliquely Propagating Waves, Physics of Fluids, 15, 2006-2013, https://doi.org/10.1063/1.1693815, 1972.

Panov, E. V., Nakamura, R., Baumjohann, W., Kubyshkina, M. G., Artemyev, A. V., Sergeev, V. A., Petrukovich, A. A., Angelopoulos, V., Glassmeier, K.-H., McFadden, J. P., and Larson, D.: Kinetic ballooning/interchange instability in a bent plasma sheet, J. Geophys. Res., 117, A06228, https://doi.org/10.1029/2011JA017496, 2012.

Panov, E. V., Artemyev, A. V., Baumjohann, W., Nakamura, R., and Angelopoulos, V.: Transient electron precipitation during oscillatory BBF braking: THEMIS observations and theoretical estimates, J. Geophys. Res., 118, 3065-3076, https://doi.org/10.1002/jgra.50203, 2013.

Pollock, C., Moore, T., Jacques, A., Burch, J., Gliese, U., Saito, Y., Omoto, T., Avanov, L., Barrie, A., Coffey, V., Dorelli, J., Gershman, D., Giles, B., Rosnack, T., Salo, C., Yokota, S., Adrian, M., Aoustin, C., Auletti, C., Aung, S., Bigio, V., Cao, N., Chandler, M., Chornay, D., Christian, K., Clark, G., Collinson, G., Corris, T., De Los Santos, A., Devlin, R., Diaz, T., Dickerson, T., Dickson, C., Diekmann, A., Diggs, F., Duncan, C., Figueroa-Vinas, A., Firman, C., Freeman, M., Galassi, N., Garcia, K., Goodhart, G., Guererro, D., Hageman, J., Hanley, J., Hemminger, E., Holland, M., Hutchins, M., James, T., Jones, W., Kreisler, S., Kujawski, J., Lavu, V., Lobell, J., LeCompte, E., Lukemire, A., MacDonald, E., Mariano, A., Mukai, T., Narayanan, K., Nguyan, Q., Onizuka, M., Paterson, W., Persyn, S., Piepgrass, B., Cheney, F., Rager, A., Raghuram, T., Ramil, A., Reichenthal, L., Rodriguez, H., Rouzaud, J., Rucker, A., Saito, Y., Samara, M., Sauvaud, J.-A., Schuster, D., Shappirio, M., Shelton, K., Sher, D., Smith, D., Smith, K., Smith, S., Steinfeld, D., Szymkiewicz, R., Tanimoto, K., Taylor, J., Tucker, C., Tull, K., Uhl, A., Vloet, J., Walpole, P., Weidner, S., White, D., Winkert, G., Yeh, P.-S., and Zeuch, M.: Fast Plasma Investigation for Magnetospheric Multiscale, Space Sci. Rev. , 199, 331-406, https://doi.org/10.1007/s11214-016-0245-4, 2016.

Runov, A., Sergeev, V. A., Baumjohann, W., Nakamura, R., Apatenkov, S., Asano, Y., Volwerk, M., Vörös, Z., Zhang, T. L., Petrukovich, A., Balogh, A., Sauvaud, J., Klecker, B., and Rème, H.: Electric current and magnetic field geometry in flapping magnetotail current sheets, Annales Geophysicae, 23, 1391-1403, 2005.

Runov, A., Sergeev, V. A., Nakamura, R., Baumjohann, W., Apatenkov, S., Asano, Y., Takada, T., Volwerk, M., Vörös, Z., Zhang, T. L., Sauvaud, J., Rème, H., and Balogh, A.: Local structure of the magnetotail current sheet: 2001 Cluster observations, Annales Geophysicae, 24, 247-262, 2006.

Runov, A., Angelopoulos, V., Sitnov, M. I., Sergeev, V. A., Bonnell, J., McFadden, J. P., Larson, D., Glassmeier, K., and Auster, U.: THEMIS observations of an earthward-propagating dipolarization front, Geophys. Res. Lett., 36, L14 106, https://doi.org/10.1029/2009GL038980, 2009.

Runov, A., Angelopoulos, V., Gabrielse, C., Zhou, X.-Z., Turner, D., and Plaschke, F.: Electron fluxes and pitch-angle distributions at dipolarization fronts: THEMIS multipoint observations, J. Geophys. Res., 118, 744-755, https://doi.org/10.1002/jgra.50121, 2013.

Runov, A., Sergeev, V. A., Angelopoulos, V., Glassmeier, K.-H., and Singer, H. J.: Diamagnetic oscillations ahead of stopped dipolarization fronts, J. Geophys. Res., 119, 1643-1657, https://doi.org/10.1002/2013JA019384, 2014.

Russell, C. T., Anderson, B. J., Baumjohann, W., Bromund, K. R., Dearborn, D., Fischer, D., Le, G., Leinweber, H. K., Leneman, D., Magnes, W., Means, J. D., Moldwin, M. B., Nakamura, R., Pierce, D., Plaschke, F., Rowe, K. M., Slavin, J. A., Strangeway, R. J., Torbert, R., Hagen, C., Jernej, I., Valavanoglou, A., and Richter, I.: The Magnetospheric Multiscale Magnetometers, Space Sci. Rev. , 199, 189-256, https://doi.org/10.1007/s11214-014-0057-3, 2016.

Sergeev, V. A., Baumjohann, W., and Shiokawa, K.: Bi-directional electron distributions associated with near-tail flux transport, Geophys. Res. Lett., 28, 3813-3816, https://doi.org/10.1029/2001GL013334, 2001. 
https://doi.org/10.5194/angeo-2020-76

Preprint. Discussion started: 27 November 2020

(c) Author(s) 2020. CC BY 4.0 License.

(c) (i)

Sharma Pyakurel, P., Shay, M. A., Haggerty, C. C., Parashar, T. N., Drake, J. F., Cassak, P. A., and Gary, S. P.: Super-Alfvénic Propagation and Damping of Reconnection Onset Signatures, Journal of Geophysical Research (Space Physics), 123, 341-349, https://doi.org/10.1002/2017JA024606, 2018.

Stasiewicz, K., Bellan, P., Chaston, C., Kletzing, C., Lysak, R., Maggs, J., Pokhotelov, O., Seyler, C., Shukla, P., Stenflo, L., Streltsov, A., and Wahlund, J.-E.: Small Scale Alfvénic Structure in the Aurora, Space Sci. Rev. , 92, 423-533, 2000.

Stawarz, J. E., Eastwood, J. P., Varsani, A., Ergun, R. E., Shay, M. A., Nakamura, R., Phan, T. D., Burch, J. L., Gershman, D. J., Giles, B. L., Goodrich, K. A., Khotyaintsev, Y. V., Lindqvist, P.-A., Russell, C. T., Strangeway, R. J., and Torbert, R. B.: Magnetospheric Multiscale analysis of intense field-aligned Poynting flux near the Earth's plasma sheet boundary, Geophys. Res. Lett., 44, 7106-7113, https://doi.org/10.1002/2017GL073685, 2017.

Stiles, G. S., Hones, Jr., E. W., Bame, S. J., and Asbridge, J. R.: Plasma sheet pressure anisotropies, J. Geophys. Res., 83, 3166-3172, https://doi.org/10.1029/JA083iA07p03166, 1978.

Tong, Y., Vasko, I., Mozer, F. S., Bale, S. D., Roth, I., Artemyev, A. V., Ergun, R., Giles, B., Lindqvist, P. A., Russell, C. T., Strangeway, R., and Torbert, R. B.: Simultaneous Multispacecraft Probing of Electron Phase Space Holes, Geophys. Res. Lett., 45, 11,513-11,519, https://doi.org/10.1029/2018GL079044, 2018.

Torbert, R. B., Russell, C. T., Magnes, W., Ergun, R. E., Lindqvist, P.-A., LeContel, O., Vaith, H., Macri, J., Myers, S., Rau, D., Needell, J., King, B., Granoff, M., Chutter, M., Dors, I., Olsson, G., Khotyaintsev, Y. V., Eriksson, A., Kletzing, C. A., Bounds, S., Anderson, B., Baumjohann, W., Steller, M., Bromund, K., Le, G., Nakamura, R., Strangeway, R. J., Leinweber, H. K., Tucker, S., Westfall, J., Fischer, D., Plaschke, F., Porter, J., and Lappalainen, K.: The FIELDS Instrument Suite on MMS: Scientific Objectives, Measurements, and Data Products, Space Sci. Rev. , 199, 105-135, https://doi.org/10.1007/s11214-014-0109-8, 2016.

Ukhorskiy, A. Y., Sitnov, M. I., Merkin, V. G., Gkioulidou, M., and Mitchell, D. G.: Ion acceleration at dipolarization fronts in the inner magnetosphere, J. Geophys. Res., 122, 3040-3054, https://doi.org/10.1002/2016JA023304, 2017.

Vasko, I. Y., Agapitov, O. V., Mozer, F. S., Artemyev, A. V., Krasnoselskikh, V. V., and Bonnell, J. W.: Diffusive scattering of electrons by electron holes around injection fronts, J. Geophys. Res., 122, 3163-3182, https://doi.org/10.1002/2016JA023337, 2017a.

Vasko, I. Y., Agapitov, O. V., Mozer, F. S., Bonnell, J. W., Artemyev, A. V., Krasnoselskikh, V. V., Reeves, G., and Hospodarsky, G.: Electron-acoustic solitons and double layers in the inner magnetosphere, Geophys. Res. Lett., 44, 4575-4583, https://doi.org/10.1002/2017GL074026, 2017 b.

Volwerk, M., Nakamura, R., Baumjohann, W., Treumann, R. A., Runov, A., Vörös, Z., Zhang, T. L., Asano, Y., Klecker, B., Richter, I., Balogh, A., and Rème, H.: A statistical study of compressional waves in the tail current sheet, J. Geophys. Res., 108, 1429, https://doi.org/10.1029/2003JA010155, 2003.

Volwerk, M., Baumjohann, W., Glassmeier, K., Nakamura, R., Zhang, T., Runov, A., Vörös, Z., Klecker, B., Treumann, R., Bogdanova, Y., Eichelberger, H., Balogh, A., and Rème, H.: Compressional waves in the Earth's neutral sheet, Annales Geophysicae, 22, 303-315, https://doi.org/10.5194/angeo-22-303-2004, 2004a.

Volwerk, M., Glassmeier, K.-H., Runov, A., Nakamura, R., Baumjohann, W., Klecker, B., Richter, I., Balogh, A., RèMe, H., and Yumoto, K.: Flow burst-induced large-scale plasma sheet oscillation, J. Geophys. Res., 109, A11208, https://doi.org/10.1029/2004JA010533, 2004b.

Walsh, A. P., Owen, C. J., Fazakerley, A. N., Forsyth, C., and Dandouras, I.: Average magnetotail electron and proton pitch angle distributions

Walsh, A. P., Fazakerley, A. N., Forsyth, C., Owen, C. J., Taylor, M. G. G. T., and Rae, I. J.: Sources of electron pitch angle anisotropy in the magnetotail plasma sheet, J. Geophys. Res., 118, 60426054, https://doi.org/10.1002/jgra.50553, 2013. 
https://doi.org/10.5194/angeo-2020-76

Preprint. Discussion started: 27 November 2020

(c) Author(s) 2020. CC BY 4.0 License.

(c) (i)

Wang, C.-P., Zaharia, S. G., Lyons, L. R., and Angelopoulos, V.: Spatial distributions of ion pitch angle anisotropy in the near-Earth magnetosphere and tail plasma sheet, J. Geophys. Res., 118, 244-255, https://doi.org/10.1029/2012JA018275, 2013.

Wang, C.-P., Fuselier, S. A., Hairston, M., Zhang, X.-j., Zou, S., Avanov, L. A., Strangeway, R. J., Ahmadi, N., and Bortnik, J.: Event Studies of $\mathrm{O}^{+}$Density Variability Within Quiet-Time Plasma Sheet, Journal of Geophysical Research (Space Physics), 124, 4168-4187, https://doi.org/10.1029/2019JA026644, 2019a.

Wang, H., Lin, Y., Wang, X., and Guo, Z.: Generation of kinetic Alfvén waves in dayside magnetopause reconnection: A 3-D global-scale hybrid simulation, Physics of Plasmas, 26, 072102, https://doi.org/10.1063/1.5092561, 2019b.

Watt, C. E. J. and Rankin, R.: Electron Trapping in Shear Alfvén Waves that Power the Aurora, Physical Review Letters, 102, 045002, https://doi.org/10.1103/PhysRevLett.102.045002, 2009.

Wendel, D. E., Khazanov, G. V., Tripathi, A. K., Singhal, R. P., and Zesta, E.: Source of the Bursty Bulk Flow Diffuse Aurora: Electrostatic Cyclotron Harmonic and Whistler Waves in the Coupling of Bursty Bulk Flows to Auroral Precipitation, Journal of Geophysical Research (Space Physics), 124, 6669-6690, https://doi.org/10.1029/2019JA026606, 2019.

530 Yushkov, E., Petrukovich, A., Artemyev, A., and Nakamura, R.: Relationship between electron field-aligned anisotropy and dawndusk magnetic field: Nine years of Cluster observations in the Earth magnetotail, J. Geophys. Res., 122, 9294-9305, https://doi.org/10.1002/2016JA023739, 2017.

Zelenyi, L., Artemyev, A., and Petrukovich, A.: Properties of Magnetic Field Fluctuations in the Earth's Magnetotail and Implications for the General Problem of Structure Formation in Hot Plasmas, Space Sci. Rev., 188, 287-310, https://doi.org/10.1007/s11214-014-0037-7, 2015.

Zelenyi, L. M., Malova, H. V., Artemyev, A. V., Popov, V. Y., and Petrukovich, A. A.: Thin current sheets in collisionless plasma: Equilibrium structure, plasma instabilities, and particle acceleration, Plasma Physics Reports, 37, 118-160, https://doi.org/10.1134/S1063780X1102005X, 2011.

Zelenyi, L. M., Neishtadt, A. I., Artemyev, A. V., Vainchtein, D. L., and Malova, H. V.: Quasiadiabatic dynamics of charged particles in a space plasma, Physics Uspekhi, 56, 347, https://doi.org/10.3367/UFNe.0183.201304b.0365, 2013.

Zhang, X., Angelopoulos, V., Artemyev, A. V., and Liu, J.: Whistler and Electron Firehose Instability Control of Electron Distributions in and Around Dipolarizing Flux Bundles, Geophys. Res. Lett., 45, 9380-9389, https://doi.org/10.1029/2018GL079613, 2018.

Zhang, X., Angelopoulos, V., Artemyev, A. V., and Liu, J.: Energy Transport by Whistler Waves Around Dipolarizing Flux Bundles, Geophys. Res. Lett., 46, 11,718-11,727, https://doi.org/10.1029/2019GL084226, 2019. 